\title{
A Lefschetz fixed-point formula for certain orbifold $\mathrm{C}^{*}$-algebras
}

\author{
Siegfried Echterhoff, Heath Emerson, and Hyun Jeong Kim*
}

\begin{abstract}
Using Poincaré duality in K-theory, we state and prove a Lefschetz fixed point formula for endomorphisms of crossed product $\mathrm{C}^{*}$-algebras $C_{0}(X) \rtimes G$ coming from covariant pairs. Here $G$ is assumed countable, $X$ a manifold, and $X \rtimes G$ cocompact and proper. The formula in question describes the graded trace of the map induced by the automorphism on K-theory of $C_{0}(X) \rtimes G$, i.e. the Lefschetz number, in terms of fixed orbits of the spatial map. Each fixed orbit contributes to the Lefschetz number by a formula involving twisted conjugacy classes of the corresponding isotropy group, and a secondary construction that associates, by way of index theory, a group character to any finite group action on a Euclidean space commuting with a given invertible matrix.
\end{abstract}

Mathematics Subject Classification (2010). 19K35, 46L80.

Keywords. Lefschetz fixed point theorem, K-theory, KK-theory, noncommutative geometry, orbifolds.

\section{Introduction}

The goal of this article is to state and prove a 'noncommutative Lefschetz formula' for a certain class of orbifold $\mathrm{C}^{*}$-algebras $A$, and for a certain class of endomorphisms $\alpha: A \rightarrow A$. The $\mathrm{C}^{*}$-algebras in question are the crossed products $A=C_{0}(X) \rtimes G$, where $X$ is a manifold and $G$ is a countable group acting co-compactly and properly on $X$. It is well known that such actions give rise to orbifolds, and that the groupoids $X \rtimes G$ are Morita equivalent to the corresponding orbifold groupoids (e.g., see [12]). The endomorphism $\alpha: A \rightarrow A$ is associated to a covariant pair $(\phi, \zeta)$, where $\phi: X \rightarrow X$ is a map and $\zeta \in \operatorname{Aut}(G)$ is a group automorphism, with $\zeta$ and $\phi$ satisfying the equivariance condition $\phi(\zeta(g) x)=g \phi(x)$. Note that this data corresponds to a self-map $\dot{\phi}: G \backslash X \rightarrow G \backslash X$ of the space of orbits, together with a coherent family of (finite) group homomorphisms, going between the isotropy groups attached to the orbits. It corresponds to an automorphism of the orbifold determined by the action of $G$ on $X$. We consider the corresponding orbifold Lefschetz number taken

${ }^{*}$ This research was supported by the EU-Network EU-NCG (Contract MRTN-CT-2006-031962) and the Deutsche Forschungsgemeinschaft (SFB 478) and by the National Science and Engineering Research Council of Canada Discovery Grant program. 
by the trace of the induced map on the K-theory of the crossed-product $\mathrm{C}^{*}$-algebra $A=C_{0}(X) \rtimes G$ :

$$
\operatorname{Lef}(\alpha):=\operatorname{trace}_{s}\left(\alpha_{*}: \mathrm{K}_{*}(A)_{\mathbb{Q}} \rightarrow \mathrm{K}_{*}(A)_{\mathbb{Q}}\right) .
$$

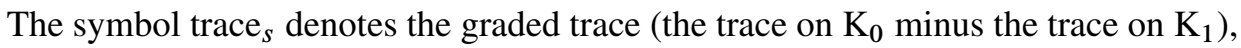
and $\alpha_{*}$ is of course the map induced on K-theory by the automorphism $\alpha: A \rightarrow A$. We aim to compute the Lefschetz number of $\alpha$ in geometric terms. Specifically, we are going to compute it in terms of 1) the fixed orbits of the spatial map of the orbit space $G \backslash X$, and 2) representation-theoretic information about the isotropy assigned to each such fixed orbit.

The geometry here is therefore in some sense the geometry of the primitive ideal space of the crossed-product $\mathrm{C}^{*}$-algebra $A$, which as a set is a bundle over $G \backslash X$ with fibre over $\dot{x} \in G \backslash X$ the irreducible dual of $\operatorname{Stab}_{G}(x)$, for any choice of $x \in \dot{x}$, but which as a topological space has multiple points at orbits with nontrivial isotropy.

If $G$ is trivial, or more generally, acts freely, then only fixed points of the induced map on the quotient $G \backslash X$ are detected, and we get essentially the classical Lefschetz fixed point theorem for $G \backslash X$.

At the other extreme, where $X$ is trivial (is a point) and, hence $G$ is finite, we just have an automorphism of a finite group. Our Lefschetz theorem then recovers the following well-known fact about automorphisms of finite groups,

$$
\#(\operatorname{Fix}(\hat{\zeta}: \hat{G} \rightarrow \widehat{G}))=\frac{1}{|G|} \sum_{g \in G}\left|Z_{\zeta}(g)\right|,
$$

where $Z_{\zeta}(g)=\{h \in G \mid \zeta(h) g=g h\}$ and $\hat{\zeta}: \hat{G} \rightarrow \widehat{G}$ is the permutation of the irreducible dual of $G$ induced by the automorphism. This theorem is often expressed in terms of 'twisted conjugacy classes' instead, the right hand side is trivially seen to be the number of $\zeta$-twisted conjugacy classes in $G$.

In the general case, the local Lefschetz data around a fixed orbit in our theorem can be described as follows: the automorphism generates a family of subgroups of the isotropy group of each fixed point, and for each such subgroup, a virtual character of that subgroup. The characters are individually averaged, and the results added up.

We now explain this in a little more detail.

Let $\rho: \Gamma \rightarrow \mathrm{O}(n, \mathbb{R})$ be an orthogonal representation of a finite group $\Gamma$, and $A \in \mathrm{GL}(n, \mathbb{R})$ a self-intertwiner of this representation, i.e., $A$ commutes with $\rho(\Gamma)$. Using this data we can define a conjugation-invariant function

$$
\chi_{(\rho, A)}: \Gamma \rightarrow \mathbb{Z}, \quad \chi_{(\rho, A)}(g)=\operatorname{sign} \operatorname{det}\left(A_{\mid \mathrm{Fix}(g)}\right),
$$

which we call the orientation character of the pair $(\rho, A)$. Of course if $g \in \Gamma$ then Fix $(g)$ is an $A$-invariant linear subspace of $\mathbb{R}^{n}$ so this makes sense.

A pleasant and apparently not entirely obvious fact is that $\chi_{(\rho, A)}$ is a virtual character, that is, a difference of characters, of the group $\Gamma$. We prove this. Indeed, 
this 'integrality result' follows from index theory. It turns out that $\chi(\rho, A)$ is the virtual character associated to the $\Gamma$-equivariant analytic index of the $\Gamma$-equivariant Schrödinger-type operator obtained by perturbing the de Rham operator $d+d^{*}$ on $L^{2}$-forms on $\mathbb{R}^{n}$ by the covector field $\mathcal{X}_{A}$, where $\mathcal{X}_{A}(x)=A x \cdot d x$.

Of course, now the fact that $\chi_{(\rho, A)}$ is a character implies that its average over the group with respect to normalized Haar measure is an integer, since by elementary representation theory this gives the dimension of the component of the trivial representation of the virtual representation corresponding to the virtual character $\chi(\rho, A)$.

Returning to the general situation of $G$ acting on $X$, choose a point $p$ from each fixed orbit of the induced map $\dot{\phi}: G \backslash X \rightarrow G \backslash X$. For each $p$ we have a secondary group action, and covariant pair, as follows.

Let $L_{p}:=\{g \in G \mid \phi(g p)=p\}$; then we have a group action of the isotropy group $\operatorname{Stab}_{G}(p)$ on $L_{p}$ by twisted conjugation $h \cdot g:=\zeta(h) g h^{-1}$. Let the orbits of this action be represented by elements $g_{1}, \ldots, g_{m}$. For each $i$, let $\Gamma_{p, i} \subset \operatorname{Stab}_{G}(p)$ be the stabilizer of $g_{i}$ under this action. One easily checks that $\Gamma_{p, i}$ commutes with $\phi \circ g_{i}$ and hence, differentiating and identifying the tangent space at $p$ with $\mathbb{R}^{n}$, one obtains a representation $\rho_{p, i}: \Gamma_{p, i} \rightarrow \mathrm{O}(n, \mathbb{R})$ and an intertwiner $A_{p, i}:=\mathrm{Id}-\left(\phi \circ g_{i}\right)^{\prime}(p)$. Then our Lefschetz theorem reads as following:

\section{Theorem 0.1. In the above notation}

$$
\operatorname{Lef}([\alpha])=\sum_{\dot{p} \in \operatorname{Fix}(\dot{\phi})} \sum_{i} \frac{1}{\left|\Gamma_{p, i}\right|} \sum_{h \in \Gamma_{p, i}} \chi_{p, i}(h),
$$

where the $\chi_{\rho_{p, i}}$ are the index characters, as in (0.1), so that

$$
\chi_{p, i}(h)=\operatorname{sign} \operatorname{det}\left(\mathrm{Id}-D_{p}\left(\phi \circ g_{i}\right)_{\left.\left.\right|_{\mathrm{Fix}(h)}\right)} .\right.
$$

The technique on which the proof of our orbifold fixed point theorem relies on is quite general, and can be phrased for general $\mathrm{C}^{*}$-algebras: we use the fact that for $\mathrm{C}^{*}$-algebras satisfying the Künneth theorem and the UCT and in addition satisfying Poincaré duality in K-theory, the Lefschetz number of an endomorphism can be phrased as an index problem. This index problem arises from the automorphism and the cycles representing the fundamental classes of the duality. More precisely, the Lefschetz number can be realized as a Kasparov product in $\operatorname{KK}(\mathbb{C}, \mathbb{C})$ : one twists the fundamental class of the Poincaré duality by the automorphism, then pair with the dual fundamental class. This index is computable in some situations by a local formula, as happens here. For more details of the general idea and an application to endomorphisms of Cuntz-Krieger algebras, see the preprint [9].

That the $\mathrm{C}^{*}$-algebras $C_{0}(X) \rtimes G$ and $C_{\tau}(X) \rtimes G$ are Poincaré dual is proved in [6]. It can be deduced from results of Kasparov on equivariant KK-theory. However, for purposes of applying the abstract Lefschetz formula of [9] we need explicit descriptions of the fundamental classes $\Delta$ and $\hat{\Delta}$. The first part of the article is devoted to finding such representatives. 
In the second part, we analyse the orientation character $\chi_{(\rho, A)}$ and in the third this becomes the critical ingredient in the computation of the appropriate index pairing, which yields the Lefschetz theorem, Theorem 0.1.

The problem of giving a good description of the K-theory of such orbifolds as appear here will be dealt with elsewhere. Such a description is needed to give a good formula for the global Lefschetz number of an automorphism. At the moment it seems to us to be a (surprisingly) delicate problem, however, and to keep down the length of the article, we have restricted our attention here to establishing the formula modulo K-theory computations with a focus on the geometric, local description of our Lefschetz invariants.

Note. All groups occurring in this article are discrete. We generally use groupalgebra notation in connection with crossed products. Thus, if $A$ is a $G$-C*-algebra, then $A \rtimes G$ is a completion of the linear span of the elements $a[g]$, with $a \in A$ and $g \in G$.

\section{Fundamental classes}

Let $X$ be a complete Riemannian manifold and let $G$ be a countable group acting isometrically, co-compactly and properly on $X$. Let $C_{\tau}(X)$ denote the algebra of continuous sections of the Clifford algebra bundle of $X$ which vanish at infinity. Since the group $G$ acts isometrically on $X$, the action extends to an action of $G$ on $C_{\tau}(X)$. We can form the crossed product $C_{\tau}(X) \rtimes G$. To fix notation, we denote by

$$
d_{g}^{x}: T_{g^{-1} x} X \rightarrow T_{x} X
$$

the differential of the action of $g$ on $X$ at the point $y=g^{-1} x$. It extends uniquely to a $*$-homomorphism $d_{g}^{x}: \operatorname{Cl}\left(T_{g^{-1}{ }_{x}} X\right) \rightarrow \operatorname{Cl}\left(T_{x} X\right)$ and the action of $G$ on the Clifford bundle $C_{\tau}(X)$ is given by

$$
g(\varphi)(x)=d_{g}^{x}\left(\varphi\left(g^{-1} x\right)\right),
$$

for $\varphi \in C_{\tau}(X), x \in X$ and $g \in G$.

In this section, we are going to first review the proof of the Poincare duality between $C_{0}(X) \rtimes G$ and $C_{\tau}(X) \rtimes G$, and then, using the proof, compute the fundamental classes for this duality. Let us first recall the following two equivalent definitions of Poincaré duality.

Definition 1.1 (cf. [6], [7]). Let $\Lambda$ and $\hat{\Lambda}$ be $C^{*}$-algebras. Then $\Lambda$ and $\hat{\Lambda}$ are Poincaré dual

(i) if there exist classes, called fundamental classes, $\Delta \in \mathrm{KK}(\Lambda \hat{\otimes} \hat{\Lambda}, \mathbb{C})$ and $\hat{\Delta} \in \operatorname{KK}(\mathbb{C}, \hat{\Lambda} \hat{\otimes} \Lambda)$ such that $\hat{\Delta} \hat{\otimes}_{\hat{\Lambda}} \Delta=1_{\Lambda}$ and $\hat{\Delta} \hat{\otimes}_{\Lambda} \Delta=1_{\hat{\Lambda}}$, or equivalently, 
(ii) if for every pair of $C^{*}$-algebras $A$ and $B$, there is an isomorphism

$$
\Phi_{A, B}: \operatorname{KK}(\Lambda \hat{\otimes} A, B) \stackrel{\cong}{\mathrm{KK}}(A, \hat{\Lambda} \hat{\otimes} B)
$$

natural with respect to intersection and composition products.

Remark 1.2. It is easy to see the equivalence of the two definitions of Poincaré duality. The isomorphism $\Phi_{A, B}$ of (ii) can be obtained by the cap product with the class $\hat{\Delta}$ over $\Lambda$ and the inverse is given by the cap product with the class $\Delta$ over $\hat{\Lambda}$. On the other hand, for a given system of isomorphisms $\left\{\Phi_{A, B}\right\}$, one can get classes $\Delta=\Phi_{\hat{\Lambda}, \mathbb{C}}^{-1}\left(1_{\hat{\Lambda}}\right)$ and $\hat{\Delta}=\Phi_{\mathbb{C}, \Lambda}\left(1_{\Lambda}\right)$.

Remark 1.3. Note that when we say $\Lambda$ and $\hat{\Lambda}$ are Poincaré dual, we already implicitly used the fact that Poincaré duality is symmetric. Indeed one can show that $\Delta^{\prime}:=$ $\sigma^{*}(\Delta) \in \operatorname{KK}(\hat{\Lambda} \hat{\otimes} \Lambda, \mathbb{C})$ and $\hat{\Delta}^{\prime}:=\sigma_{*}(\hat{\Delta}) \in \operatorname{KK}(\mathbb{C}, \Lambda \hat{\otimes} \hat{\Lambda})$ satisfy condition (i) in Definition 1.1, where $\sigma$ is the flip isomorphism.

Note 1.4. Under these circumstances, the maps

$$
\Delta_{*}: \mathrm{K}_{*}(\Lambda) \rightarrow \mathrm{K}^{*}(\hat{\Lambda}), \quad x \mapsto \hat{x}:=\left(x \otimes 1_{\hat{\Lambda}}\right) \otimes_{\Lambda \otimes \hat{\Lambda}} \Delta
$$

and

$$
\hat{\Delta}_{*}: \mathrm{K}^{*}(\hat{\Lambda}) \rightarrow \mathrm{K}_{*}(\Lambda), \quad y \mapsto \hat{y}:=\hat{\Delta} \otimes_{\hat{\Lambda} \otimes \Lambda}\left(y \hat{\otimes} 1_{\Lambda}\right)
$$

are inverse isomorphisms. Similarly, the maps

$$
\Delta^{*}: \mathrm{K}_{*}(\hat{\Lambda}) \rightarrow \mathrm{K}^{*}(\Lambda), \quad x \mapsto \hat{x}:=\left(1_{\Lambda} \otimes x\right) \otimes_{\Lambda \otimes \hat{\Lambda}} \Delta
$$

and

$$
\hat{\Delta}^{*}: \mathrm{K}^{*}(\Lambda) \rightarrow \mathrm{K}_{*}(\hat{\Lambda}), \quad y \mapsto \hat{y}:=\hat{\Delta} \otimes_{\hat{\Lambda} \otimes \Lambda}\left(1_{\hat{\Lambda}} \hat{\otimes} y\right)
$$

are inverse isomorphisms.

Recall that Kasparov duality (see [10] for an extensive discussion, or the original source [16], Theorem 4.9) states that, in this situation, and more generally where $G$ is allowed to be locally compact, there is a canonical isomorphism

$$
K_{A, B}: \operatorname{RKK}^{G}(X ; A, B) \stackrel{\cong}{\cong} \mathrm{KK}^{G}\left(C_{\tau}(X) \hat{\otimes} A, B\right)
$$

for any $G-C^{*}$-algebras $A$ and $B$. If $G$ is discrete, then for $A$ and $B$ equipped with the trivial $G$-action, we have the following canonical isomorphism

$$
\begin{aligned}
C_{A, B}: \mathrm{KK}^{G}\left(C_{\tau}(X) \hat{\otimes} A, B\right) & \cong \mathrm{\cong K}\left(\left(C_{\tau}(X) \rtimes G\right) \hat{\otimes} A, B\right), \\
{[(\mathcal{E}, \varphi, F)] } & \longmapsto[(\mathcal{E}, \varphi \rtimes \pi, F)],
\end{aligned}
$$


where $\pi$ is the group representation on $\mathcal{E}$. Moreover, if such $G$ acts properly on $X$, then, as a consequence of [18], Theorem 5.4, we have an isomorphism

$$
E_{A, B}: \operatorname{RKK}^{G}(X ; A, B) \stackrel{\cong}{\mathrm{KK}}\left(A,\left(C_{0}(X) \rtimes G\right) \hat{\otimes} B\right) .
$$

Combining all the isomorphisms above, we have Poincaré duality between $C_{0}(X) \rtimes G$ and $C_{\tau}(X) \rtimes G$ as follows: for all $C^{*}$-algebras $A$ and $B$ with trivial $G$-action, there exists an isomorphism

$$
\begin{aligned}
\Phi_{A, B}: \mathrm{KK}\left(\left(C_{\tau}(X) \rtimes G\right) \hat{\otimes} A, B\right) & \stackrel{C_{A, B}^{-1}}{\longrightarrow} \mathrm{KK}^{G}\left(C_{\tau}(X) \hat{\otimes} A, B\right) \\
& \stackrel{K_{A, B}^{-1}}{\longrightarrow} \operatorname{RKK}^{G}(X ; A, B) \\
& \stackrel{E_{A, B}}{\longrightarrow} \mathrm{KK}\left(A,\left(C_{0}(X) \rtimes G\right) \hat{\otimes} B\right),
\end{aligned}
$$

which is natural with respect to intersection and external products.

Now using the above system of isomorphisms $\left\{\Phi_{A, B}\right\}$ and the equivalence of the two definitions of Poincaré duality (see Definition 1.1 and Remark 1.2) as well as the symmetry of Poincaré duality (Remark 1.3), we can compute fundamental classes

$$
\Delta=\sigma^{*}\left(\Phi_{C_{0}(X) \rtimes G, \mathbb{C}}^{-1}\left(1_{C_{0}(X) \rtimes G}\right)\right) \text { and } \hat{\Delta}=\sigma_{*}\left(\Phi_{\mathbb{C}, C_{\tau}(X) \rtimes G}\left(1_{C_{\tau}(X) \rtimes G}\right)\right) .
$$

For explicit descriptions for $\Delta$ and $\hat{\Delta}$, we need an extensive discussion on the map $\Phi_{A, B}$, i.e., the maps $C_{A, B}, K_{A, B}$ and $E_{A, B}$. We already know the map $C_{A, B}$. The map $K_{A, B}$ is the isomorphism of Kasparov's first Poincare duality. Recall the following Remark 1.5 and Lemma 1.6 from [16].

Remark 1.5. (i) Let $d: L^{2}\left(\Lambda_{\mathbb{C}}^{*} X\right) \rightarrow L^{2}\left(\Lambda_{\mathbb{C}}^{*} X\right)$ denote the (densely defined) de Rham operator. Let $D=d+d^{*}$ and let $F$ be the pseudodifferential operator $D\left(1+D^{2}\right)^{-\frac{1}{2}}$. Then $\left(L^{2}\left(\Lambda_{\mathbb{C}}^{*} X\right), F\right)$ is a cycle for an element in $\mathrm{KK}^{G}\left(C_{\tau}(X), \mathbb{C}\right)$ where the action of $C_{\tau}(X)$ on $L^{2}\left(\Lambda_{\mathbb{C}}^{*} X\right)$ comes from the identification as vector bundles of the Clifford bundle of $X$ and the exterior bundle. We denote this cycle by $[D]$.

(ii) The map

$$
\sigma_{X, C_{\tau}(X)}: \operatorname{RKK}^{G}(X ; A, B) \rightarrow \mathrm{KK}^{G}\left(C_{\tau}(X) \hat{\otimes} A, C_{\tau}(X) \hat{\otimes} B\right)
$$

associates to a cycle $(\mathcal{E}, F)$ for $\mathrm{RKK}^{G}(X ; A, B)$ the cycle $\left(C_{\tau}(X) \hat{\otimes}_{C_{0}(X)} \mathcal{E}, 1 \hat{\otimes} F\right)$ for $\mathrm{KK}^{G}\left(C_{\tau}(X) \hat{\otimes} A, C_{\tau}(X) \hat{\otimes} B\right)$. The map $\sigma_{X, C_{\tau}(X)}$ is natural with respect to intersection products in the sense that

$$
\sigma_{X, C_{\tau}(X)}\left(f \hat{\otimes}_{B} f^{\prime}\right)=\sigma_{X, C_{\tau}(X)}(f) \hat{\otimes}_{C_{\tau}(X) \hat{\otimes} B} \sigma_{X}\left(f^{\prime}\right),
$$

for $f \in \mathrm{RKK}^{G}(X ; A, B), f^{\prime} \in \mathrm{RKK}^{G}(X ; B, C)$. 
(iii) At the level of cycles, the map

$$
p_{X}^{*}: \mathrm{KK}^{G}(A, B) \rightarrow \mathrm{RKK}^{G}(X ; A, B)
$$

tensors with the standard representative of $1_{C_{0}(X)} \in \mathrm{KK}^{G}\left(C_{0}(X), C_{0}(X)\right)$. Note that $p_{X}^{*}$ is natural with respect to intersection products in the sense that $p_{X}^{*}\left(f_{1} \hat{\otimes}_{B} f_{2}\right)=$ $\left(p_{X}^{*}\left(f_{1}\right)\right) \hat{\otimes}_{X, B}\left(p_{X}^{*}\left(f_{2}\right)\right)$ for $f_{1} \in \mathrm{KK}^{G}(A, B), f_{2} \in \mathrm{KK}^{G}(B, C)$.

(iv) Let $\rho$ denote the metric on $X$. Then there exists an open neighbourhood $U$ of the diagonal in $X \times X$ where for every point $(x, y) \in U$ there exists a unique geodesic from $x$ to $y$. For such $U$ let $\mathcal{F}_{U}$ be the ideal of $C_{0}(X) \hat{\otimes} C_{\tau}(X)$ of Clifford sections supported on $U$. Since $G$ acts isometrically and cocompactly on $X$, there exists $\varepsilon>0$ such that $U_{\varepsilon}:=\{(x, y) \mid \rho(x, y)<\varepsilon\}$ is contained in the set $U$. Let $\theta_{\varepsilon}(x, y)=$ $\frac{\rho(x, y)}{\varepsilon}\left(d_{y} \rho\right)(x, y)$. Then $\left(\mathscr{F}_{U_{\varepsilon}}, \theta_{\varepsilon}\right)$ defines a cycle in $\operatorname{RKK}^{G}\left(X ; \mathbb{C}, C_{\tau}(X)\right)$ with $\theta_{\varepsilon}$ as a multiplicative operator and $\left[\left(\widetilde{F}_{U_{\varepsilon}}, \theta_{\varepsilon}\right)\right]=\left[\left(\widetilde{F}_{\varepsilon_{\varepsilon^{\prime}}}, \theta_{\varepsilon^{\prime}}\right)\right]$ for any $0<\varepsilon^{\prime} \leq \varepsilon$. We denote the class $\left[\left(\mathcal{F}_{U_{\varepsilon}}, \theta_{\varepsilon}\right)\right]$ by $\Theta$, and we shall simply write $\left(\mathscr{F}_{U}, \theta\right)$ if we do not want to specify the special $\varepsilon$ used in the construction. A slightly more complicated construction yields a similar class $\Theta=\left[\left(\mathscr{F}_{U}, \theta\right)\right]$ for possibly non-cocompact isometric actions.

The following is a special case of Kasparov's [16], Theorem 4.9:

Lemma 1.6. Let $G$ act isometrically on a complete Riemannian manifold $X$. The composition

$$
\begin{gathered}
K_{A, B}: \operatorname{RKK}^{G}(X ; A, B) \stackrel{\sigma_{X, C_{\tau}(X)}}{\longrightarrow} \mathrm{KK}^{G}\left(C_{\tau}(X) \hat{\otimes} A, C_{\tau}(X) \hat{\otimes} B\right) \\
\stackrel{-\otimes[D]}{\longrightarrow} \mathrm{KK}^{G}\left(C_{\tau}(X) \hat{\otimes} A, B\right)
\end{gathered}
$$

is an isomorphism with inverse the composition

$$
\begin{aligned}
K_{A, B}^{-1}: \mathrm{KK}^{G}\left(C_{\tau}(X) \hat{\otimes} A, B\right) & \stackrel{p_{X}^{*}}{\longrightarrow} \operatorname{RKK}^{G}\left(X ; C_{\tau}(X) \hat{\otimes} A, B\right) \\
& \stackrel{\Theta \widehat{\otimes}-}{\longrightarrow} \operatorname{RKK}^{G}(X ; A, B) .
\end{aligned}
$$

The map $E_{A, B}$ is the isomorphism from [18], Theorem 5.4. To understand the map $E_{A, B}$ explicitly, we need to understand two ingredients. Firstly, the descent homomorphism

$$
\text { descent: } \operatorname{RKK}^{G}(X ; A, B) \rightarrow \mathrm{KK}\left(C_{0}(X, A) \rtimes G, C_{0}(X, B) \rtimes G\right) .
$$

Secondly, the unit class $[E] \in \mathrm{K}_{0}\left(C_{0}(X) \rtimes G\right)$, defined whenever $G \backslash X$ is compact: $[E]$ is defined by the finitely generated projective right $C_{0}(X) \rtimes G$-module $E$ which is the completion of $C_{c}(X)$ with respect to the inner product

$$
\left\langle\varphi, \varphi^{\prime}\right\rangle(x, g)=\varphi(x) \varphi^{\prime}(g x) .
$$


For future reference, the right action of $C_{0}(X) \rtimes G$ on $E$ is given by

$$
\varphi f(x)=\varphi(x) f(x), \quad \varphi g(x)=\varphi(g x), \quad g \in G, f \in C_{0}(X) .
$$

Remark 1.7. $[E]$ is also represented by the projection $P \in C_{0}(X) \rtimes G$,

$$
P=\sum_{g \in G} \varphi g(\varphi)
$$

where $\varphi \in C_{c}(X)$ is chosen so that $0 \leq \varphi \leq 1, \sum_{g \in G} g(\varphi)^{2}=1$. See [4].

Lemma 1.8 ([18], Theorem 5.4; [11], Lemma 20). Let $G$ act properly and cocompactly on $X$. The map $E_{A, B}: \operatorname{RKK}^{G}(X ; A, B) \rightarrow \operatorname{KK}\left(A,\left(C_{0}(X) \rtimes G\right) \hat{\otimes} B\right)$ given by the composition

$$
\begin{aligned}
& \operatorname{RKK}^{G}(X ; A, B) \stackrel{\text { descent }}{\longrightarrow} \\
& \mathrm{KK}\left(C_{0}(X, A) \rtimes G, C_{0}(X, B) \rtimes G\right) \\
& \stackrel{[E] \otimes-}{\longrightarrow} \mathrm{KK}\left(A,\left(C_{0}(X) \rtimes G\right) \hat{\otimes} B\right)
\end{aligned}
$$

is an isomorphism whenever $A$ and $B$ are $G$-trivial $C^{*}$-algebras.

Remark 1.9 (cf. [18]). The map $E_{A, B}$ can be explicitly described as follows. Suppose that we have a cycle $(\varepsilon, F)$ for $\operatorname{RKK}^{G}(X ; A, B)$. Then $\varepsilon$ is a right $C_{0}(X, B)$-module, and a left $C_{0}(X, A)$-module, and the two actions of $C_{0}(X)$ on the left and right agree. Furthermore, the group $G$ acts on $\mathcal{E}$. We can assume by averaging that $F$ is exactly $G$-invariant. Now we complete the compactly supported elements of $\mathscr{E}$ to a right $C_{0}(X, B) \rtimes G$-module $\tilde{\mathscr{E}}$ using the inner product valued in $C_{0}(X, B) \rtimes G$,

$$
\left\langle\xi, \xi^{\prime}\right\rangle=\sum_{h \in G}\left\langle\xi, h\left(\xi^{\prime}\right)\right\rangle[h] .
$$

The right action of $C_{0}(X, A) \rtimes G$ is given by letting $C_{0}(X, A)$ act as originally, and $G$ acting by $\xi h=h^{-1}(\xi)$.

Finally, we note that adjointable operators on the right $C_{0}(X, B) \rtimes G$-module $\tilde{\mathcal{E}}$ are in 1-1-correspondence with $G$-equivariant operators on $\mathcal{E}$. (Generalizations of the isomorphism $E_{A, B}$ are given in [11], [6].)

Now we are going to construct fundamental classes $\Delta$ and $\hat{\Delta}$. First, we construct the class $\Delta$. Recall Remark 1.5 (i) for the discussion below. We define two commuting $*$-homomorphisms $C_{\tau}(X) \rtimes G \rightarrow \mathbb{B}\left(L^{2}\left(\Lambda_{\mathbb{C}}^{*} X\right) \hat{\otimes} \ell^{2} G\right)$ and $C_{0}(X) \rtimes G \rightarrow$ $\mathbb{B}\left(L^{2}\left(\Lambda_{\mathbb{C}}^{*} X\right) \hat{\otimes} \ell^{2} G\right)$, by constructing two corresponding covariant pairs. We let $e_{g} \in \ell^{2} G$ denote the point-mass at $g \in G$.

The $*$-homomorphism $C_{\tau}(X) \rtimes G \rightarrow \mathbb{B}\left(L^{2}\left(\Lambda_{\mathbb{C}}^{*} X\right) \hat{\otimes} \ell^{2} G\right)$ is defined by the covariant pair

$$
\varphi\left(\xi \hat{\otimes} e_{g}\right)=\varphi \cdot \xi \hat{\otimes} e_{g}, \quad h\left(\xi \hat{\otimes} e_{g}\right)=h \cdot \xi \hat{\otimes} e_{g h^{-1}}
$$


for $g, h \in G, \xi \in L^{2}\left(\Lambda_{\mathbb{C}}^{*} X\right), \varphi \in C_{\tau}(X)$. The $*$-homomorphism $C_{0}(X) \rtimes G \rightarrow$ $\mathbb{B}\left(L^{2}\left(\Lambda_{\mathbb{C}}^{*} X\right) \hat{\otimes} \ell^{2} G\right)$ is defined by the covariant pair

$$
f\left(\xi \hat{\otimes} e_{g}\right)=g^{-1}(f) \cdot \xi \hat{\otimes} e_{g}, \quad h\left(\xi \hat{\otimes} e_{g}\right)=\xi \hat{\otimes} e_{h g}
$$

for $f \in C_{0}(X), g, h \in G, \xi \in L^{2}\left(\Lambda_{\mathbb{C}}^{*} X\right)$. The dots indicate the actions already implied in the cycle $D=\left(L^{2}\left(\Lambda_{\mathbb{C}}^{*} X\right), F\right)$ of Kasparov (see Remark 1.5 (i)); note that $C_{0}(X)$ embeds in $C_{\tau}(X)$. Observe that the two $*$-homomorphisms just defined commute, and so determine a $*$-homomorphism

$$
\Pi: C_{0}(X) \rtimes G \hat{\otimes} C_{\tau}(X) \rtimes G \rightarrow \mathbb{B}\left(L^{2}\left(\Lambda_{\mathbb{C}}^{*} X\right) \hat{\otimes} \ell^{2} G\right) .
$$

Let $g \in G, f \in C_{0}(X)$ and $\varphi \in C_{\tau}(X)$ be compactly supported. If $T$ is a locally compact operator on $L^{2}\left(\Lambda_{\mathbb{C}}^{*} X\right)$, e.g., if $T=F^{2}-1$, then

$$
(f \hat{\otimes} \varphi)(T \hat{\otimes} 1)\left(\xi \hat{\otimes} e_{g}\right)=g^{-1}(f) \varphi T \xi \hat{\otimes} e_{g},
$$

so that $(f \hat{\otimes} \varphi)(T \hat{\otimes} 1)$ acts as the block diagonal operator $\bigoplus_{g \in G}\left(g^{-1}(f) \varphi T\right) \hat{\otimes} 1$, which has compact blocks. As

$$
g^{-1}(f) \varphi=0 \quad \text { for } g \notin H:=\left\{h \in G \mid h^{-1}(\operatorname{supp}(f)) \cap \operatorname{supp}(\varphi) \neq \emptyset\right\},
$$

since the indicated set $H$ is finite and since $G$ acts properly, there are only finitely many blocks. Thus $(f \hat{\otimes} \varphi)\left(\left(F^{2}-1\right) \hat{\otimes} 1\right)$ is compact. This observation and similar ones prove that the Hilbert space $L^{2}\left(\Lambda_{\mathbb{C}}^{*} X\right) \hat{\otimes} \ell^{2} G$ equipped with the $*$-homomorphism $\Pi: C_{\tau}(X) \rtimes G \hat{\otimes} C_{0}(X) \rtimes G \rightarrow \mathbb{B}\left(L^{2}\left(\Lambda_{\mathbb{C}}^{*} X\right) \hat{\otimes} \ell^{2} G\right)$ defined above and the operator $F \hat{\otimes} 1$ defines a cycle for $\operatorname{KK}\left(C_{0}(X) \rtimes G \widehat{\otimes} C_{\tau}(X) \rtimes G, \mathbb{C}\right)$.

Definition 1.10. We define

$$
\Delta \in \mathrm{KK}\left(C_{0}(X) \rtimes G \hat{\otimes} C_{\tau}(X) \rtimes G, \mathbb{C}\right)
$$

to be the class of the cycle $\left(L^{2}\left(\Lambda_{\mathbb{C}}^{*} X\right) \hat{\otimes} \ell^{2} G, \Pi, F \hat{\otimes} 1\right)$ above.

We shall show below that $\Delta$ coincides with the fundamental class of (1.2).

The dual class $\hat{\Delta}$ is more complicated to write down. Recall the class $\Theta=$ $\left[\left(\mathcal{F}_{U}, \theta\right)\right]$ from Remark 1.5 (iv). We consider the completion $\&$ of $C_{c}(X) \mathscr{F}_{U} \otimes \widehat{\mathbb{C}} G$ (with action of $C_{c}(X)$ with respect to the first variable in $U \subseteq X \times X$ ) equipped with the following $C_{\tau}(X) \rtimes G \hat{\otimes} C_{0}(X) \rtimes G$-valued inner product:

$$
\left\langle\alpha \hat{\otimes}[g], \alpha^{\prime} \hat{\otimes}\left[g^{\prime}\right]\right\rangle=\sum_{h \in G} g^{-1}\left(\alpha^{*} h\left(\alpha^{\prime}\right)\right)\left[g^{-1} h g^{\prime}\right] \hat{\otimes}\left[g^{-1} h\right] .
$$

Here $\left[g^{-1} h\right]$ is understood as in $G \subset C_{0}(X) \rtimes G,\left[g^{-1} h g^{\prime}\right]$ is understood as in $G \subset C_{\tau}(X) \rtimes G$ and $g^{-1}\left(\alpha^{*} h\left(\alpha^{\prime}\right)\right)$ is understood as in $C_{\tau}(X) \hat{\otimes} C_{0}(X)$, equipped with the diagonal $G$-action. The right module-structure is given by

$$
\begin{array}{rlrl}
(\alpha \hat{\otimes}[g]) f & =f \alpha \hat{\otimes}[g], & & (\alpha \hat{\otimes}[g]) h=h^{-1}(\alpha) \hat{\otimes}\left[h^{-1} g\right], \\
(\alpha \hat{\otimes}[g]) \varphi=\alpha g(\varphi) \hat{\otimes}[g], & (\alpha \hat{\otimes}[g]) h^{\prime}=\alpha \hat{\otimes}\left[g h^{\prime}\right],
\end{array}
$$


where $f \in C_{0}(X), h \in G \subset C_{0}(X) \rtimes G$ and $\varphi \in C_{\tau}(X), h^{\prime} \in G \subset C_{\tau}(X) \rtimes G$.

Note that any $G$-invariant element of $M\left(C_{\tau}(X) \hat{\otimes} C_{0}(X)\right)$ acts as an operator on $\mathscr{E}$ by multiplication in the $\mathscr{F}_{U}$-variable. The $G$-invariance is needed to commute with the action of $G \subset C_{0}(X) \rtimes G$ on the right.

Definition 1.11. The class $\hat{\Delta} \in \mathrm{KK}\left(\mathbb{C}, C_{\tau}(X) \rtimes G \hat{\otimes} C_{0}(X) \rtimes G\right)$ is given by the pair $\left(\mathcal{E}, \theta_{G}\right)$, where we let $\theta_{G}$ be the operator on $\mathcal{E}$ induced by the $G$-invariant multiplier $\theta$ of $\mathscr{F}_{U} \subset C_{0}(X) \hat{\otimes} C_{\tau}(X)$ as described in Remark 1.5 (iv), and $\mathcal{E}$ is the Hilbert module as above with the inner product and the right actions given in (1.8) and (1.9).

Now we need to prove that the classes $\Delta$ and $\hat{\Delta}$ defined above are actually the fundamental classes for $\Lambda=C_{0}(X) \rtimes G$ and $\hat{\Lambda}=C_{\tau}(X) \rtimes G$, i.e., they satisfy identities $\Delta=\sigma^{*}\left(\Phi_{C_{0}(X) \rtimes G, \mathbb{C}}^{-1}\left(1_{C_{0}(X) \rtimes G}\right)\right) \in \operatorname{KK}(\Lambda \hat{\otimes} \hat{\Lambda}, \mathbb{C})$ and $\hat{\Delta}=$ $\left.\sigma_{*}\left(\Phi_{\mathbb{C}, C_{\tau}(X) \rtimes G}\left(1_{C_{\tau}(X) \rtimes G}\right)\right) \in \operatorname{KK}(\mathbb{C}, \hat{\Lambda} \hat{\otimes} \Lambda)\right)$ from (1.2), where $\sigma$ denotes the flip isomorphism. This will follow from

Proposition 1.12. Let $\Delta$ and $\hat{\Delta}$ be the classes defined in Definitions 1.10 and 1.11 and let $\Phi_{(-,-)}$be the isomorphisms (1.1). Then

$$
\sigma_{*}\left(\Phi_{\mathbb{C}, C_{\tau}(X) \rtimes G}\left(1_{C_{\tau}(X) \rtimes G}\right)\right)=\hat{\Delta} \quad \text { and } \quad \Phi_{C_{0}(X) \rtimes G, \mathbb{C}}\left(\sigma^{*} \Delta\right)=1_{C_{0}(X) \rtimes G} .
$$

For the proof we need some preliminary discussion.

Definition and Remark 1.13. We give - here and elsewhere - the crossed product $C_{0}(X) \rtimes G$ the structure of a trivial $G-C^{*}$-algebra. Let $A$ be an $X \rtimes G$-algebra. The multiplication class $m_{A}$ is the class

$$
m_{A} \in \mathcal{R K K}^{G}\left(X ; A \hat{\otimes} C_{0}(X) \rtimes G, A\right)
$$

given by the cycle $\left(A \hat{\otimes} \ell^{2} G, 0\right)$, where the right Hilbert $A$-structure of $A \hat{\otimes} \ell^{2} G$ is the obvious one, and the further module structures are as follows.

Note that there are two $G$ 's involved here; one the $G$ which appears in $\mathcal{R K K}^{G}$, the other which appears in the crossed product $C_{0}(X) \rtimes G$. To reduce confusion, we refer to the action of the former as the equivariant action. The equivariant action of $G$ on $A \hat{\otimes} \ell^{2} G$ is then given by $h\left(a \hat{\otimes} e_{g}\right)=h(a) \hat{\otimes} e_{g h^{-1}}$. The $C_{0}(X)$-structure is by multiplication in the $A$ factor.

The representation of the crossed product is given by the covariant pair

$$
f\left(a \hat{\otimes} e_{g}\right)=g^{-1}(f) a \hat{\otimes} e_{g}, \quad h\left(a \hat{\otimes} e_{g}\right)=a \hat{\otimes} e_{h g} .
$$

One easily checks that this is a covariant pair. The left actions of $C_{0}(X) \rtimes G$ and of $C_{0}(X)$ clearly commute, and the $*$-homomorphism $C_{0}(X) \rtimes G \rightarrow \mathbb{B}\left(A \hat{\otimes} \ell^{2} G\right)$ is equivariant, that is, $C_{0}(X) \rtimes G$ acts as $G$-invariant operators on $A \hat{\otimes} \ell^{2} G$. Since the action of $A \hat{\otimes} C_{0}(X) \rtimes G$ is by compact operators on $A \hat{\otimes} \ell^{2} G$ we get a cycle as required. 
Lemma 1.14. The isomorphism

$$
E_{C_{0}(X) \rtimes G, \mathbb{C}}: \operatorname{RKK}^{G}\left(X ; C_{0}(X) \rtimes G, \mathbb{C}\right) \rightarrow \operatorname{KK}\left(C_{0}(X) \rtimes G, C_{0}(X) \rtimes G\right)
$$

maps $m_{C_{0}(X)}$ to $1_{C_{0}(X) \rtimes G}$.

Proof. Apply the explicit description in Remark 1.9. We get the completion of $C_{0}(X) \hat{\otimes} \mathbb{C} G$ with respect to the inner product

$$
\left\langle a \hat{\otimes} e_{g}, a^{\prime} \hat{\otimes} e_{g^{\prime}}\right\rangle=a^{*} g^{-1}\left(g^{\prime}\left(a^{\prime}\right)\right)\left[g^{-1} g^{\prime}\right] \in C_{0}(X) \rtimes G
$$

for $a, a^{\prime} \in C_{0}(X), g, g^{\prime} \in G$, and the right $C_{0}(X) \rtimes G$-module structure

$$
\left(a \hat{\otimes} e_{g}\right) f=f a \hat{\otimes} e_{g}, \quad\left(a \hat{\otimes} e_{g}\right) h=h^{-1}(a) \hat{\otimes} e_{g h} .
$$

The left action of $C_{0}(X) \rtimes G$ is given by

$$
f\left(a \hat{\otimes} e_{g}\right)=g^{-1}(f) a \hat{\otimes} e_{g}, \quad h\left(a \hat{\otimes} e_{g}\right)=a \hat{\otimes} e_{h g} .
$$

Let $\overline{C_{0}(X) \hat{\otimes} \ell^{2} G}$ be the completion of the above to a Hilbert module. We define a map $W: \overline{C_{0}(X) \hat{\otimes} \ell^{2} G} \rightarrow C_{0}(X) \rtimes G$, where the co-domain has its standard $C_{0}(X) \rtimes G$-bimodule structure, by the formula

$$
W\left(a \hat{\otimes} e_{g}\right)=g(a)[g] .
$$

Then

$$
\left\langle W^{-1}(a[g]), W^{-1}\left(a^{\prime}\left[g^{\prime}\right]\right)\right\rangle=\left\langle g^{-1}(a) \hat{\otimes} e_{g},\left(g^{\prime}\right)^{-1}\left(a^{\prime}\right) \hat{\otimes} e_{g^{\prime}}\right\rangle=g^{-1}\left(a^{*} a^{\prime}\right)\left[g^{-1} g^{\prime}\right],
$$

and

$$
\begin{aligned}
W^{-1}(a[g] h) & =W^{-1}(a[g h])=h^{-1}\left(g^{-1}(a)\right) \hat{\otimes} e_{g h}=\left(g^{-1}(a) \hat{\otimes} e_{g}\right) h, \\
W^{-1}(a[g] f) & =W^{-1}(a g(f)[g])=g^{-1}(a) f \hat{\otimes} e_{g}=\left(W^{-1}(a[g])\right) f .
\end{aligned}
$$

Hence $W$ gives an isometry between the inner product we have defined initially on $C_{0}(X) \hat{\otimes} \mathbb{C} G$, and the usual inner product on the free, rank one Hilbert $C_{0}(X) \rtimes G$ module.

Similarly, one checks that $W$ conjugates the left $C_{0}(X) \rtimes G$-module structure we have defined above and the standard one by algebra multiplication.

Therefore the image of the class $m_{C_{0}(X)}$ under the map $E_{C_{0}(X) \rtimes G, \mathbb{C}}$ sends the cycle for the multiplication class to a cycle which is unitarily equivalent to the standard representative of $1_{C_{0}(X)}$, so that $E_{C_{0}(X) \rtimes G, \mathbb{C}}\left(m_{C_{0}(X)}\right)=1_{C_{0}(X)}$ as claimed.

Proof of Proposition 1.12. Consider first the fundamental class $\Delta$. This is accomplished by a direct computation: by Lemma 1.14, it remains to apply the map from Remark 1.5 (ii),

$$
\sigma_{X, C_{\tau}(X)}: \operatorname{RKK}^{G}\left(X ; C_{0}(X) \rtimes G, \mathbb{C}\right) \rightarrow \mathrm{KK}^{G}\left(C_{\tau}(X) \hat{\otimes} C_{0}(X) \rtimes G, C_{\tau}(X)\right),
$$


to the class $m_{C_{0}(X)}$. A straightforward application of the definition gives the cycle $\left(C_{\tau}(X) \hat{\otimes} \ell^{2} G, 0\right)$. The left action of $C_{\tau}(X)$ is given by $\varphi\left(a \hat{\otimes} e_{g}\right)=\varphi a \hat{\otimes} e_{g}$, the group $G$ acts by $h\left(a \hat{\otimes} e_{g}\right)=h(a) \hat{\otimes} e_{g h^{-1}}$. The left action of $C_{0}(X) \rtimes G$ is given by the covariant pair

$$
f\left(a \hat{\otimes} e_{g}\right)=g^{-1}(f) a \hat{\otimes} e_{g}, \quad h\left(a \hat{\otimes} e_{g}\right)=a \hat{\otimes} e_{h g} .
$$

Finally, we take the product of the class of this cycle, with the class $D \in$ $\mathrm{KK}^{G}\left(C_{\tau}(X), \mathbb{C}\right)$ of Kasparov (see Remark $1.5(\mathrm{i})$ ). Comparing with Definition 1.10, we see that the modules are the same. The axioms for a Kasparov product imply that the operator involved in the product is also that described in Definition 1.10.

The assertion regarding $\hat{\Delta}$ is similar but slightly more straightforward; we leave its confirmation to the reader.

\section{The orientation character}

Before proceeding to the Lefschetz theorem, we need to perform an index calculation on Euclidean space $\mathbb{R}^{n}$ generalising the computation of the index of the Schrödinger operator $\frac{d}{d x} \pm x$ on $L^{2}(\mathbb{R})$ (see [13]). The analogue for $\mathbb{R}^{n}$ of the harmonic oscillator in dimension 1 is the operator $D+\mathcal{X}$, where $D=d+d^{*}$ is the de Rham operator acting on $L^{2}\left(\Lambda_{\mathbb{C}}^{*} \mathbb{R}^{n}\right)$, and $\mathcal{X}$ is Clifford multiplication by the form $x_{1} d x_{1}+\cdots+x_{n} d x_{n}$ on $\mathbb{R}^{n}$. The (unbounded) cycle $\left(L^{2}\left(\Lambda_{\mathbb{C}}^{*} \mathbb{R}^{n}\right), D+\mathcal{X}\right)$ represents the Kasparov product

$$
[X] \hat{\otimes}_{C_{\tau}\left(\mathbb{R}^{n}\right)}[D] \in \mathrm{KK}^{\mathrm{O}(n, \mathbb{R})}(\mathbb{C}, \mathbb{C})=\mathrm{R}(\mathrm{O}(n, \mathbb{R}))
$$

of the class $[D]$ of the de Rham operator (see Remark 1.5) the generator $[X] \in$ $\mathrm{KK}^{\mathrm{O}(n, \mathbb{R})}\left(\mathbb{C}, C_{\tau}\left(\mathbb{R}^{n}\right)\right)$ constructed via the covector field $\mathcal{X}$ as explained below (by identifying $\mathcal{X}$ with the vector field $x \mapsto x$ on $\left.\mathbb{R}^{n}\right)$. It is the content of Kasparov's Bottperiodicity theorem $[15]$, Theorem 7 of $\S 5$, that $[X] \widehat{\otimes}_{C_{\tau}\left(\mathbb{R}^{n}\right)}[D]=1 \in \mathrm{R}(\mathrm{O}(n, \mathbb{R}))$.

In this section we extend Kasparov's calculations to the following more general situation: we assume that $\Gamma$ is a compact group acting on $\mathbb{R}^{n}$ via an orthogonal representation $\rho: \Gamma \rightarrow \mathrm{O}(n, \mathbb{R})$. Moreover, we shall assume that $A \in \operatorname{GL}(n, \mathbb{R})$ commutes with $\rho$. Let $\mathcal{X}_{A}$ denote the covector field $x \mapsto A x \cdot d x$ acting on $L^{2}\left(\Lambda_{\mathbb{C}}^{*} \mathbb{R}^{n}\right)$ via Clifford multiplication. We then obtain a $\Gamma$-invariant Fredholm operator $\mathcal{X}_{A}+D$ (or a bounded version of it) and we need to compute the $\Gamma$-index

$$
\operatorname{ind}^{\Gamma}\left(X_{A}+D\right) \in \mathrm{R}(\Gamma) .
$$

We shall do this in two different ways: in a first version we make use of Kasparov's ideas for the proof of his Bott-periodicity theorem by reducing the computations to appropriate two- and one-dimensional subspaces. In a second version we sketch the argument how the result can also be obtained from a use of the Atiyah-Singer Index Theorem for open subsets of $\mathbb{R}^{N}$ (see [1]) together with some calculations given by Atiyah and Segal in [2]. 
Before we do this we need to recall the relation between vector fields on a manifold $X$ and corresponding classes in $K_{0}^{\Gamma}\left(C_{\tau}(X)\right)$. So let $X$ be any Riemannian manifold such that the compact group $\Gamma$ acts isometrically on $X$. Suppose that $v: X \rightarrow T X$ is a $\Gamma$-invariant continuous vector field on $X$ such that there exists a compact set $K \subseteq X$ with $v(x) \neq 0$ outside $K$. We then say that $v$ is co-compactly supported. From $v$ we construct a new vector field $\tilde{v}$ as follows: choose a $\Gamma$-invariant positive continuous function $\varphi: X \rightarrow[0,1]$ such that $\varphi \equiv 0$ on $K$ and such that $1-\varphi \in C_{0}(X)$. Then set

$$
\tilde{v}(x):=\varphi(x) \frac{v(x)}{\|v(x)\|} .
$$

The vector field $\tilde{v}$ acts as self-adjoint bounded operator on $C_{\tau}(X)$ by point-wise Clifford multiplication such that $\tilde{v}^{2}-1$ (which is point-wise multiplication by $x \mapsto$ $\left.\left(\|\tilde{v}(x)\|^{2}-1\right)\right)$ lies in $C_{\tau}(X)$. It thus defines a class $[v] \in \mathrm{KK}^{\Gamma}\left(\mathbb{C}, C_{\tau}(X)\right)=$ $K_{0}^{\Gamma}\left(C_{\tau}(X)\right)$.

Two such vector fields $v_{0}, v_{1}: X \rightarrow T X$ are said to be homotopic, if there exists a co-compactly supported $\Gamma$-invariant continuous map $v: X \times[0,1] \rightarrow T X$ such that $v(x, t) \in T_{x} X$ for all $(x, t) \in X \times[0,1]$ and

$$
\left.v\right|_{X \times\{0\}}=v_{0} \quad \text { and }\left.\quad v\right|_{X \times\{1\}}=v_{1} .
$$

Lemma 2.1. Suppose that $v: X \rightarrow T X$ is a co-compactly supported $\Gamma$-invariant continuous vector field. Then the class $[v] \in \mathrm{K}_{0}^{\Gamma}\left(C_{\tau}(X)\right)$ does not depend on the choice of the function $\varphi$. Moreover, two homotopic co-compactly supported vector fields on $X$ determine the same class in $\mathrm{K}_{0}^{\Gamma}\left(C_{\tau}(X)\right)$.

Proof. Suppose that $v(x) \neq 0$ outside the compact set $K \subseteq X$ and suppose that $\varphi_{0}$ and $\varphi_{1}$ are two functions which vanish on $K$ and which have value 1 at $\infty$. Then

$$
t \mapsto \tilde{v}_{t}=\left(t \varphi_{1}+(1-t) \varphi_{0}\right) \frac{v}{\|v\|}
$$

is an operator homotopy between $\tilde{v}_{0}$ and $\tilde{v}_{1}$ which proves the first assertion. A similar argument gives the second assertion.

Recall from Remark 1.5 the construction of the Dirac class $[D]=\left[D_{X}\right] \in$ $\mathrm{KK}_{0}^{\Gamma}\left(C_{\tau}(X), \mathbb{C}\right)$ given by the de Rham operator $D=d+d^{*}: L^{2}\left(\Lambda_{\mathbb{C}}^{*}(X)\right) \rightarrow$ $L^{2}\left(\Lambda_{\mathbb{C}}^{*}(X)\right)$. Note that if $U \subseteq X$ is any open $\Gamma$-invariant sub-manifold, then $\left[D_{X}\right]$ restricts to the class $\left[D_{U}\right]$ under the canonical inclusion $\iota_{U}: C_{\tau}(U) \rightarrow C_{\tau}(X)$. The following basic (and certainly well-known) lemma turns out to be extremely useful for our computations.

Lemma 2.2. Suppose that $v: X \rightarrow T X$ is a co-compactly supported $\Gamma$-invariant vector field on $X$. Let $K \subseteq X$ be compact such that $v$ does not vanish outside $K$ and let $U \subseteq X$ be an open $\Gamma$-invariant neighborhood of $K$ in $X$. Then

$$
\left[v_{U}\right] \otimes_{C_{\tau}(U)}\left[D_{U}\right]=[v] \otimes_{C_{\tau}(X)}[D] \in \mathrm{R}(\Gamma),
$$

where $v_{U}: U \rightarrow T U$ denotes the restriction of $v$ to $U$. 
Proof. By the construction of the class $[v]$ we may assume without loss of generality that there exists a compact $\Gamma$-invariant set $C \subseteq U$ such that $\|v(x)\|=1$ for all $x \notin C$. The Kasparov product $[v] \otimes_{C_{\tau}(X)}[D]$ is represented by the pair

$$
\left(L^{2}\left(\Lambda_{\mathbb{C}}^{*}(X)\right), T\right) \quad \text { with } T=\lambda_{v(x)}+\lambda_{v(x)}^{*}+\sqrt{1-\|v(x)\|^{2}} \frac{D}{\sqrt{1+D^{2}}}
$$

and with $D=d+d^{*}$, which can be deduced from [15], Remark 3 on p. 541 . Since $\|v(x)\|^{2}=1$ outside $U$, it follows that the second summand vanishes on $X \backslash U$. It is then clear that $L^{2}\left(\Lambda_{\mathbb{C}}^{*}(X)\right)$ decomposes into the direct product of $T$ invariant subspaces $L^{2}\left(\Lambda_{\mathbb{C}}^{*}(U)\right) \oplus L^{2}\left(\Lambda_{\mathbb{C}}^{*}(X \backslash U)\right)$ such that the restriction of $T$ to $L^{2}\left(\Lambda_{\mathbb{C}}^{*}(U)\right)$ gives the product $\left[v_{U}\right] \otimes_{C_{\tau}(U)}\left[D_{U}\right]$ (since $D$ is local). The restriction of $T$ to $L^{2}\left(\Lambda_{\mathbb{C}}^{*}(X \backslash U)\right)$ is given point-wise by the unitary operator $\lambda_{v(x)}+\lambda_{v(x)}^{*}$ (it is unitary since $\|v(x)\|=1$ ) and hence has index 0 .

Remark 2.3. Suppose that $\left(\varepsilon_{1}, \phi_{1}, F_{1}\right)$ and $\left(\varepsilon_{2}, \phi_{2}, F_{2}\right)$ are two Kasparov cycles giving elements $x \in \mathrm{KK}^{G}(A, B)$ and $y \in \mathrm{KK}^{G}(B, C)$, respectively, where we assume here that $G$ is a compact group. Assume that both operators $F_{1}, F_{2}$ are $G$ invariant and self-adjoint with $\left\|F_{1}\right\| \leq 1$. Suppose further that $F \in \mathbb{B}\left(E_{1} \hat{\otimes}_{B} \varepsilon_{2}\right)$ is a self-adjoint $F_{2}$-connection, i.e.,

$$
\Theta_{\xi} F_{2}-(-1)^{\operatorname{deg}(\xi) \cdot \operatorname{deg}\left(F_{2}\right)} F \Theta_{\xi} \in \mathbb{K}\left(\mathcal{E}_{2}, \mathcal{E}_{1} \widehat{\otimes}_{B} \varepsilon_{2}\right)
$$

for all $\xi \in \mathcal{E}_{1}$, where $\Theta_{\xi}: \mathcal{E}_{2} \rightarrow \mathcal{E}_{1} \hat{\otimes}_{B} \mathcal{E}_{2} ; \eta \mapsto \xi \hat{\otimes}_{B} \eta$. Let

$$
T=\left(F_{1} \otimes 1\right)+\left(1-F_{1}^{2} \otimes 1\right)^{1 / 2} F \in \mathbb{B}\left(\mathcal{E}_{1} \hat{\otimes}_{B} \varepsilon_{2}\right) .
$$

It then follows from [5], 18.10.1, that $\left(\mathcal{E}_{1} \otimes_{B} \mathcal{E}_{2}, \phi_{1} \otimes 1, T\right)$ is a representative for the Kasparov product $x \hat{\otimes}_{B} y \in \mathrm{KK}^{G}(A, C)$, provided that $\left[T, \phi_{1}(A) \hat{\otimes} 1\right] \epsilon$ $\mathbb{K}\left(\mathcal{E}_{1} \hat{\otimes}_{B} \mathcal{E}_{2}\right)$. Formula (2.1) is a direct consequence of this principle. But we shall use this principle also in a more advanced setting in $\S 3$ below.

We now specialize to the case where $X=V$ is a finite dimensional Euclidean vector space together with a linear action $\rho: \Gamma \rightarrow \mathrm{O}(V)$. We want to give explicit computations of the product $[v] \otimes_{C_{\tau}(V)}\left[D_{V}\right]$ in the case where $v: V \rightarrow T V=V \times V$ is given by $v(x)=A x$ for some $A \in \mathrm{GL}(V)$ which commutes with the representation $\rho$. We shall always write $\mathcal{X}_{A}$ for this vector field. We shall show below that the product $\left[\mathcal{X}_{A}\right] \otimes_{C_{\tau}(V)}[D] \in \mathrm{KK}^{\Gamma}(\mathbb{C}, \mathbb{C})=\mathrm{R}(\Gamma)$ is equal to the orientation character $\chi_{(\rho, A)}$ as in

Definition 2.4. Let $\rho: \Gamma \rightarrow \mathrm{O}(V)$ and $A \in \mathrm{GL}(V)$ as above. The orientation character $\chi_{(\rho, A)}: \Gamma \rightarrow \mathbb{Z}$ is the conjugation-invariant function on $\Gamma$

$$
\chi_{(\rho, A)}(g):=\operatorname{sign} \operatorname{det}\left(A_{\left.\right|_{\mathrm{Fix}(g)}}\right),
$$

where $\operatorname{Fix}(g) \subseteq V$ denotes the space of fixed-points for $g \in \Gamma$. 
The set Fix $(g)$ is of course a linear subspace of $V$ invariant under $A$, so the formula makes sense. It is clear that $\chi_{(\rho, A)}$ is conjugation-invariant. The remaining part of this section is devoted to the proof of

Theorem 2.5. The orientation character $\chi_{(\rho, A)}$ is a virtual character of $\Gamma$ (i.e., a difference of two characters). Under the identification of $\mathrm{R}(\Gamma)$ as the ring of $\mathbb{Z}$ linear combinations of characters of $\Gamma$, we have

$$
\chi_{(\rho, A)}=\left[\mathcal{X}_{A}\right] \otimes_{C_{\tau}(V)}[D] .
$$

Remark 2.6. (a) Recall that the identification of $R(\Gamma)$ with the ring of $\mathbb{Z}$-linear combinations of characters of $\Gamma$ is given by sending a finite dimensional representation $\pi: \Gamma \rightarrow \operatorname{End}(\mathcal{H})$ to its character $\chi_{\pi}(g)=\operatorname{trace}(\pi(g))$ (the $n o n$-normalized trace on $\operatorname{End}(\mathscr{H})$ ). If a class in $\mathrm{KK}^{\Gamma}(\mathbb{C}, \mathbb{C})=\mathrm{R}(\Gamma)$ is represented by a $\Gamma$-invariant Fredholm operator $F: \mathscr{H}^{\text {even }} \rightarrow \mathscr{H}^{\text {odd }}$, then the corresponding virtual character in $\mathrm{R}(\Gamma)$ is given by the difference function $\chi_{-} \chi_{+}-\chi_{-}$, where $\chi_{+}$and $\chi_{-}$denote the traces of the $\Gamma$-representations on $\mathscr{H}_{+}=\operatorname{ker}(F)$ and $\mathscr{H}_{-}=\operatorname{coker}(F)$, respectively. Since the value at a point $g \in \Gamma$ only depends on the action of $g$ on these spaces, it follows that in order to compute it we may always restrict our attention to the closed subgroup $\Gamma_{g} \subseteq \mathrm{O}(V)$ generated by $\rho(g)$.

Recall also that the identification $\mathrm{KK}^{\Gamma}(\mathbb{C}, \mathbb{C}) \cong \mathrm{R}(\Gamma)$ is multiplicative in the sense that it sends the Kasparov product $\widehat{\otimes} \mathbb{C}$ on $\mathrm{KK}^{\Gamma}(\mathbb{C}, \mathbb{C})$ to the pointwise product of characters in $\mathrm{R}(\Gamma)$.

(b) We may always assume that $A \in \mathrm{O}(V)$. Indeed, if $A=O|A|$ is the polar decomposition of $A$ with $O=A|A|^{-1}$, then the homotopy $t \mapsto O(t \operatorname{Id}+(1-t)|A|)$ between $A$ and $O$ induces a $\Gamma$-invariant homotopy between the vector fields $X_{A}$ and $O X$, and the result follows from Lemma 2.1.

(c) In case where $A=\mathrm{Id}$ is the identity, we obtain the class $[X] \in \mathrm{K}_{0}^{\Gamma}\left(C_{\tau}(V)\right)$. It is the "canonical" generator of $\mathrm{K}_{0}^{\Gamma}\left(C_{\tau}(V)\right)$ as described by Kasparov in [15], § 5, and it follows from Kasparov's Bott-periodicity theorem [15], Theorem 7 of $\S 5$, that $[X] \otimes_{C_{\tau}(V)}[D]=1 \in \mathrm{R}(\Gamma)$ (in the language of [15], the class $[X]$ is denoted $\beta_{V}$ and $[D]$ is denoted $\left.\alpha_{V}\right)$.

Note that in case of the trivial group $\Gamma=\{e\}$ the above theorem reduces to an index computation given by Lück and Rosenberg in [20].

The quantity we will be interested in for our Lefschetz theorem is the component of the trivial representation in $\chi_{(\rho, A)}$ : this is obtained by averaging the character over $\Gamma$; thus we derive the formula

Corollary 2.7. Suppose that $F: \mathscr{H}^{\text {even }} \rightarrow \mathscr{H}^{\text {odd }}$ is a $\Gamma$-equivariant Fredholm operator representing the Kasparov product $\left[\mathcal{X}_{A}\right] \otimes_{C_{\tau}(V)}[D] \in \mathrm{R}(\Gamma)$. Then

$$
\operatorname{dim}_{\mathbb{C}}\left(\operatorname{ker}^{\Gamma} F\right)-\operatorname{dim}_{\mathbb{C}}\left(\operatorname{coker}^{\Gamma} F\right)=\int_{\Gamma} \chi_{(\rho, A)}(g) d g
$$

(normalized Haar measure) where $V^{\Gamma}$ denotes the $\Gamma$-fixed points of a $\Gamma$-module $V$. 
Example 2.8. In this example we want to compute the class $\left[\mathcal{X}_{A}\right] \hat{\otimes}_{C_{\tau}(\mathbb{R})}\left[D_{\mathbb{R}}\right] \in \mathrm{R}(\Gamma)$ in the special case where $V=\mathbb{R}$ is one-dimensional, following the lines of Kasparov's [14], Example 3 on p. 760. This gives the key calculation for the proof of Theorem 2.5. By part (b) and (c) of the above remark we may assume that $A$ is multiplication by -1 . Also, by Lemma 2.2 we may restrict everything to the interval $(-\pi, \pi)$. If we identify $L^{2}\left(\Lambda_{\mathbb{C}}^{0}(-\pi, \pi)\right)$ and $L^{2}\left(\Lambda_{\mathbb{C}}^{1}(-\pi, \pi)\right)$ with $L^{2}(-\pi, \pi)$ in the canonical way, we can realize the class $D=D_{(-\pi, \pi)}$ by the matrix $D=\left(\begin{array}{cc}0 & d / d x \\ -d / d x & 0\end{array}\right)$. On the basis $\left\{e_{n} \mid n \in \mathbb{Z}\right\}$ with $e_{n}(x)=e^{i n x}$ the operator $\frac{d}{d x}$ acts by $e_{n} \mapsto i n e_{n}$, thus we obtain a bounded version $\tilde{d}: L^{2}(-\pi, \pi) \rightarrow L^{2}(-\pi, \pi)$ of the operator $d=\frac{d}{d x}$ by defining

$$
\tilde{d} e_{n}=i \operatorname{sign}(n) e_{n} \quad \text { with } \operatorname{sign}(n)= \begin{cases}0 & \text { if } n=0, \\ \frac{n}{|n|} & \text { if } n \neq 0 .\end{cases}
$$

The vector field $x \mapsto-x$ on $(-\pi, \pi)$ is homotopic to $x \mapsto-\sin \left(\frac{x}{2}\right)$. Thus, using Lemma 2.2 and the formula for the Kasparov product as given in (2.1) it follows that $[-\mathcal{X}] \hat{\otimes}_{C_{\tau}(\mathbb{R})}\left[D_{\mathbb{R}}\right]$ is given by the $\Gamma$-equivariant index of the operator

$$
T:=-\sin \left(\frac{x}{2}\right)+\cos \left(\frac{x}{2}\right) \tilde{d}: L^{2}(-\pi, \pi) \rightarrow L^{2}(-\pi, \pi) .
$$

To compute it we first calculate the index of the operator

$$
S:=2 i e^{i \frac{x}{2}} T=\left(1-e^{i x}\right)+i\left(e^{i x}+1\right) \tilde{d},
$$

which in terms of the orthonormal basis $\left\{e_{n} \mid n \in \mathbb{Z}\right\}$ is given by

$$
S e_{n}= \begin{cases}-2 e_{n+1} & \text { if } n>0 \\ e_{0}-e_{1} & \text { if } n=0 \\ 2 e_{n} & \text { if } n<0\end{cases}
$$

It then follows from a short computation that $\operatorname{ker} S=\{0\}$ and coker $S=\left\langle e_{0}+e_{1}\right\rangle$. Going back to the original operator $T$ we get $\operatorname{ker} T=\{0\}$ and coker $T$ is generated by $\frac{1}{2} e^{-i \frac{x}{2}}\left(1+e^{i x}\right)=\cos \left(\frac{x}{2}\right)$.

If we write $\mathrm{O}(\mathbb{R})=\{1,-1\}$, then the corresponding action of -1 on $L^{2}(-\pi, \pi) \cong$ $L^{2}\left(\Lambda_{\mathbb{C}}^{1}(-\pi, \pi)\right)$ is given by $\xi \mapsto(x \mapsto-\xi(-x))$. Thus, on the generator $\xi(x)=$ $\cos \left(\frac{x}{2}\right)$ of coker $T$ it acts by multiplication with -1 . It follows that $[-X] \hat{\otimes}_{C_{\tau}(\mathbb{R})}$ $\left[D_{\mathbb{R}}\right] \in \mathrm{R}(\Gamma)$ is represented by the virtual character $\chi$ given by

$$
\chi(g)= \begin{cases}-1 & \text { if } \rho(g)=1, \\ 1 & \text { if } \rho(g)=-1 .\end{cases}
$$

The following lemma will allow to reduce the proof of Theorem 2.5 to the case of the above example. 
Lemma 2.9. For $i=1,2$ let $V_{i}$ be an Euclidean vector space with representation $\rho_{i}: \Gamma \rightarrow O\left(V_{i}\right)$ and let $A_{i} \in \mathrm{GL}\left(V_{i}\right)$ commute with $\rho_{i}$. Let $V=V_{1} \oplus V_{2}, \rho=\rho_{1} \oplus \rho_{2}$ and $A=A_{1} \oplus A_{2}$. Then

$$
\left[\mathcal{X}_{A}\right] \hat{\otimes}_{C_{\tau}(V)}\left[D_{V}\right]=\left(\left[\mathcal{X}_{A_{1}}\right] \hat{\otimes}_{C_{\tau}\left(V_{1}\right)}\left[D_{V_{1}}\right]\right) \cdot\left(\left[\mathcal{X}_{A_{2}}\right] \hat{\otimes}_{C_{\tau}\left(V_{2}\right)}\left[D_{V_{2}}\right]\right) \in \mathrm{R}(\Gamma) .
$$

Proof. It is not difficult to check that under the canonical isomorphism $C_{\tau}(V) \cong$ $C_{\tau}\left(V_{1}\right) \hat{\otimes} C_{\tau}\left(V_{2}\right)$ we get $\left[\mathcal{X}_{A}\right]=\left[\mathcal{X}_{A_{1}}\right] \hat{\otimes}_{\mathbb{C}}\left[\mathcal{X}_{A_{2}}\right]$ in $\mathrm{KK}^{\Gamma}\left(C_{\tau}(V), \mathbb{C}\right)$ (compare with the formula for $\beta_{V}$ in [15], p. 546) and it is shown in [15], p. 547, that $\left[D_{V}\right]=$ $\left[D_{V_{1}}\right] \widehat{\otimes}_{\mathbb{C}}\left[D_{V_{2}}\right]$ in $\mathrm{KK}^{\Gamma}\left(\mathbb{C}, C_{\tau}(V)\right)$. The result then follows from the associativity of the Kasparov product.

Proof of Theorem 2.5. Let $g \in \Gamma$ be fixed. As observed in Remark 2.6 we may assume that $\Gamma=\Gamma_{g}$ is the closed subgroup of $\mathrm{O}(V)$ generated by $\rho(g)$ (which we then identify with $g$ ). We also observed that we may assume without loss of generality that $A \in \mathrm{O}(V)$. Let $F \subseteq V$ be the set of $g$-fixed-points in $V$ and let $N=F^{\perp}$. Then $F$ and $N$ are both, $g$ - and $A$-invariant, and therefore the result will follow from the above lemma if we can show that

$$
\left(\left[X_{A_{F}}\right] \hat{\otimes}_{C_{\tau}(F)}\left[D_{F}\right]\right)(g)=\operatorname{sign} \operatorname{det}\left(A_{F}\right),
$$

where $A_{F}$ denotes the restriction of $A$ to $F$, and

$$
\left(\left[\mathcal{X}_{A_{N}}\right] \hat{\otimes}_{C_{\tau}(F)}\left[D_{N}\right]\right)(g)=1 .
$$

Since $\Gamma_{g}$ acts trivially on $F$, we may choose an orthonormal basis $\left\{v_{1}, \ldots, v_{l}\right\}$ of $F$ and, up to homotopy, we may assume that $A_{F}$ is given with respect to this basis by $\left(\begin{array}{cc} \pm 1 & 0 \\ 0 & I_{l-1}\end{array}\right)$. If the upper left entry is 1 we have $\left[\mathcal{X}_{A_{F}}\right]=[X]$ and the result follows from Kasparov's Bott-periodicity theorem (see Remark 2.6 (c)). If the upper left entry is -1 , we apply the above lemma to the decomposition $F=\left\langle v_{1}\right\rangle \oplus\left\langle v_{2}, \ldots, v_{l}\right\rangle$. Since $A_{F}$ restricts to the identity on $\left\langle v_{2}, \ldots, v_{l}\right\rangle$, this summand provides the factor +1 to the character at $g$ and since $g$ acts trivially on $\left\langle v_{1}\right\rangle$ it follows from Example 2.8 that the first summand provides the factor -1 to the character at $g$. This verifies (2.2).

To verify (2.3) we first consider the -1 eigenspace $V_{-1}$ for the action of $g$ on $N$. This is clearly $\Gamma_{g}$ - and $A$-invariant, and we may consider the decomposition $N=V_{-1} \oplus V_{-1}^{\perp}$ of $N$ as in the lemma. If $B$ denotes the restriction of $A$ to $V_{-1}$ we may again assume, up to $\Gamma_{g}$-invariant homotopy, that $B=\left(\begin{array}{cc} \pm 1 & 0 \\ 0 & I_{k-1}\end{array}\right)$ with respect to a suitable orthonormal base $\left\{w_{1}, \ldots, w_{k}\right\}$ of $V_{-1}$. Decomposing

$$
V_{-1}=\left\langle w_{1}\right\rangle \oplus\left\langle w_{2}, \ldots, w_{k}\right\rangle
$$

the second summand provides the factor 1 by Bott-periodicity and the summand $\left\langle w_{1}\right\rangle$ provides also the factor 1 by Example 2.8, since $g$ acts via the flip on $\mathbb{R} w_{1}$.

We therefore may assume without loss of generality that the action of $g$ on $N$ does not have eigenvalues 1 or -1 . If $N \neq\{0\}$ let $\lambda_{t}=\cos (t)+i \sin (t)$ be a complex 
eigenvalue for the action of $g$ on the complexification $N_{\mathbb{C}}=N+i N$ of $N$ and let $V_{t} \subseteq N_{\mathbb{C}}$ denote the corresponding eigenspace. Again, since $A$ commutes with $g$, it follows that $V_{t}$ is $A$-invariant. Since $A$ is orthogonal (and hence it acts unitarily on the complex vector space $V_{t}$ ) there exists a non-zero $A$-eigenvector $u=u_{1}+i u_{2} \in V_{t}$ for some eigenvalue $\lambda_{s}=\cos (s)+i \sin (s), s \in[0,2 \pi)$. It follows then from basic linear algebra that if we choose $u$ to be a unit vector in $N_{\mathbb{C}}$, then $\sqrt{2} u_{1}, \sqrt{2} u_{2}$ are orthogonal unit vectors in $N$ and then $g$ and $A$ act on the invariant subspace $\left\langle u_{1}, u_{2}\right\rangle \subseteq N$ via rotation by the angles $t$ and $s$, respectively. But then we can $\Gamma_{g^{-}}$ equivariantly homotop the restriction of $A$ to $\left\langle u_{1}, u_{2}\right\rangle$ to the identity, which shows that the direct summand $\left\langle u_{1}, u_{2}\right\rangle$ provides the factor 1 to the character at $g$. Equation (2.3) now follows from a straightforward induction argument.

In the remaining part of this section we want to discuss briefly how Theorem 2.5 can also be obtained by appealing to Atiyah and Singer [1]. For ease of notation let $V=\mathbb{R}^{n}$ with standard inner product. The cycle $\left(L^{2}\left(\Lambda_{\mathbb{C}}^{*} \mathbb{R}^{n}\right), D+X_{A}\right)$ is an unbounded representative for the Kasparov product of the classes $[X] \otimes \mathcal{C}_{\tau}\left(\mathbb{R}^{n}\right)[D]$ (see [17], Lemma 4, and also [3] and [19] for the realization of KK-classes by unbounded operators), it therefore is a $\Gamma$-equivariant Fredholm operator on $L^{2}\left(\Lambda_{\mathbb{C}}^{*}\left(\mathbb{R}^{n}\right)\right)$ and has a $\Gamma$-equivariant index $\operatorname{ind}_{\mathrm{a}}^{\Gamma}(D+\mathcal{X}) \in \mathrm{R}(\Gamma)$ such that

$$
\operatorname{ind}_{\mathrm{a}}^{\Gamma}(D+\mathcal{X})=[\mathcal{X}] \hat{\otimes}_{C_{\tau}\left(\mathbb{R}^{n}\right)}[D] \in \mathrm{R}(\Gamma) .
$$

We now eliminate Clifford algebras from the picture, using the tangent bundle instead, using the well known $\mathrm{KK}^{\Gamma}$-equivalence between $C_{\tau}\left(\mathbb{R}^{n}\right)$ and $C_{0}\left(T \mathbb{R}^{n}\right)$ (a consequence of Kasparov's Bott-periodicity - see [15], §5, Theorem 8, [5], 24.5). Under this equivalence $[D]$ becomes the class $[\not D]$ of the Dolbeault operator on $T \mathbb{R}^{n} \cong \mathbb{C}^{n}$, and $[X]$ becomes in the notation of Atiyah-Singer the Bott generator, $j_{0} !(1) \in \mathrm{KK}^{\Gamma}\left(\mathbb{C}, C_{0}\left(T \mathbb{R}^{n}\right)\right)=\mathrm{K}_{\Gamma}^{0}\left(T \mathbb{R}^{n}\right)$, where $j_{0}:\{0\} \rightarrow \mathbb{R}^{n}$ is the inclusion of the origin of $\mathbb{R}^{n}$. Atiyah and Singer say the index map takes the class $j_{0}$ !(1) to 1 . On the other hand, the class $\left[\mathcal{X}_{A}\right] \in \mathrm{K}_{0}^{\Gamma}\left(C_{\tau}\left(\mathbb{R}^{n}\right)\right)$ corresponds to $A_{*}\left(j_{0} !(1)\right) \in \mathrm{K}_{\Gamma}^{0}\left(T \mathbb{R}^{n}\right)$. Therefore, following [1], computing $\left[\mathcal{X}_{A}\right] \widehat{\otimes}_{C_{\tau}\left(\mathbb{R}^{n}\right)}[D]=\operatorname{ind}_{\mathrm{a}}^{\Gamma}\left(D+\mathcal{X}_{A}\right)$ is equivalent to computing the topological index $\operatorname{ind}_{\mathrm{t}}^{\Gamma}\left(A_{*}\left(j_{0} !(1)\right)\right) \in \mathrm{R}(\Gamma)$ as introduced in [1].

As before, in order to compute the character at $g$ it suffices to assume that $\Gamma=\Gamma_{g}$ is the subgroup of $\mathrm{O}(n, \mathbb{R})$ generated by $\rho(g)$. For convenience, let $\beta_{W} \in \mathrm{O}(n, \mathbb{R})(T W)$ be the Bott generator, whenever $W$ is a $\Gamma$-invariant linear subspace of $\mathbb{R}^{n}$. As is well known, $\mathrm{O}(n, \mathbb{R})(T W)$ is a rank-one $\mathrm{R}(\Gamma)$-module with generator $\beta_{W}$. Equivariant Bott periodicity ind $\mathrm{t}_{\mathrm{t}}^{\Gamma}: \mathrm{O}(n, \mathbb{R})(T W) \rightarrow \mathrm{R}(\Gamma)$ commutes with the module action, and $\operatorname{ind}_{\mathrm{t}}^{\Gamma}\left(\beta_{W}\right)=1 \in \mathrm{R}(\Gamma)$ by Atiyah-Singer [1]. To be explicit, let $\sigma: \pi^{*} E \rightarrow \pi^{*} E$ be an odd endomorphism of $\mathbb{Z} / 2$-graded bundles, with $\sigma$ an isomorphism outside of a compact subset of $T \mathbb{R}^{n}$, and so representing a class $a \in \mathrm{O}(n, \mathbb{R})\left(T \mathbb{R}^{n}\right)$. Suppose $b \in \mathrm{R}(\Gamma)$ is represented by a finite-dimensional $\Gamma$-vector space $V$. Then $a \cdot b$ is represented by $\sigma \otimes \operatorname{Id}_{V}: \pi^{*}(E \otimes V) \rightarrow \pi^{*}(E \otimes V)$. 
The cycle for $\mathrm{O}(n, \mathbb{R})\left(T \mathbb{R}^{n}\right)$ representing $A_{*} \beta_{\mathbb{R}^{n}}$ is given by the trivial $\mathbb{Z} / 2$-graded bundle $T \mathbb{R}^{n} \times \Lambda_{\mathbb{C}}^{*}\left(\mathbb{R}^{n}\right)$ together with the odd endomorphism $\sigma: T \mathbb{R}^{n} \times \Lambda_{\mathbb{C}}^{*}\left(\mathbb{R}^{n}\right) \rightarrow$ $T \mathbb{R}^{n} \times \Lambda_{\mathbb{C}}^{*}\left(\mathbb{R}^{n}\right)$ determined by the map $T \mathbb{R}^{n} \rightarrow \mathbb{C}^{n},(x, \xi) \mapsto A x+i \xi$ (using Clifford multiplication.) Note that as $A x+i \xi$ vanishes only at the origin of $T \mathbb{R}^{n}$, the endomorphism $\sigma$ is an isomorphism outside of a compact set.

Let $F$ denote the fixed subspace of $g$ and $N=F^{\perp}$. We have a well-known isomorphism

$$
\Lambda_{\mathbb{C}}^{*}\left(\mathbb{R}^{n}\right) \cong \Lambda_{\mathbb{C}}^{*}(F) \hat{\otimes} \Lambda_{\mathbb{C}}^{*}(N)
$$

of graded vector spaces, and there is a corresponding isomorphism of (trivial) bundles. Note that $F$ and $N$ are also $A$-invariant.

If we restrict $\sigma: T \mathbb{R}^{n} \times \Lambda_{\mathbb{C}}^{*}\left(\mathbb{R}^{n}\right) \rightarrow T \mathbb{R}^{n} \times \Lambda_{\mathbb{C}}^{*}\left(\mathbb{R}^{n}\right)$ to $T F$, then under the identification (2.4), the endomorphism $\sigma$, when restricted to $T F$, becomes the endomorphism $\sigma \hat{\otimes} \operatorname{Id}_{N}: T F \times \Lambda_{\mathbb{C}}^{*}(F) \hat{\otimes} \Lambda_{\mathbb{C}}^{*}(N) \rightarrow T F \times \Lambda_{\mathbb{C}}^{*}(F) \hat{\otimes} \Lambda_{\mathbb{C}}^{*}(N)$. Thus we have the following.

Lemma 2.10. If $i_{F}: F \rightarrow \mathbb{R}^{n}$ is the $\Gamma$-equivariant inclusion, then

$$
i_{F}^{*}\left(A_{*}\left(\beta_{\mathbb{R}^{n}}\right)\right)=\operatorname{sign} \operatorname{det}\left(A_{\mid F}\right) \beta_{F} \cdot\left[\Lambda_{\mathbb{C}}^{*}(N)\right] \in \mathrm{O}(n, \mathbb{R})(T F),
$$

where $\Lambda_{\mathbb{C}}^{*}(N) \in \mathrm{R}(\Gamma)$ is given by $\sum_{i=0}^{\operatorname{dim}(N)}(-1)^{i}\left[\Lambda_{\mathbb{C}}^{i} N\right]$, an alternating sum of finite-dimensional $\Gamma$-spaces.

Proof. Given the preceding discussion, it is clear that

$$
i_{F}^{*}\left(A_{*}\left(\beta_{\mathbb{R}^{n}}\right)\right)=\left(A_{\mid F}\right)_{*}\left(\beta_{F}\right) \cdot\left[\Lambda_{\mathbb{C}}^{*}(N)\right] \in \mathrm{O}(n, \mathbb{R})(T F) .
$$

Since $\Gamma$ acts trivially on $F$ (and $T F$ ), if the restriction of $A$ to $F$ has positive determinant, it is $\Gamma$-equivariantly homotopic to the identity. If the determinant is negative, it is similarly homotopic to a reflection $Q$, and it is standard that $Q_{*}\left(\beta_{F}\right)=$ $-\beta_{F}$ in non-equivariant $\mathrm{K}$-theory, but then in this case also, because the $\Gamma$-action on $T F$ is trivial.

Following a pattern of argumentation in Atiyah-Segal [2], since $\rho(g)$ the generator of $\Gamma$ has no fixed points in $N$, the class $\left[\Lambda_{\mathbb{C}}^{*}(N)\right]$ is a unit in the localization $\mathrm{R}(\Gamma)_{g}$ of the ring $\mathrm{R}(\Gamma)$ at the prime ideal determined by $g$ (see [2], Lemma 2.7) - indeed, this prime ideal consists of all characters which vanish at $g$, while the character corresponding to $\left[\Lambda_{\mathbb{C}}^{*}(N)\right]$ is

$$
g \mapsto \sum_{i=0}^{\operatorname{dim}(N)}(-1)^{i} \operatorname{trace}\left(g: \Lambda_{\mathbb{C}}^{i} N \rightarrow \Lambda_{\mathbb{C}}^{i} N\right)=\operatorname{det}\left(1-g_{\left.\right|_{N}}\right) \neq 0 .
$$

For this reason and the above calculation, it follows that $i_{F}^{*}: \mathrm{O}(n, \mathbb{R})\left(T \mathbb{R}^{n}\right) \rightarrow$ $\mathrm{O}(n, \mathbb{R})(T F)$ is an isomorphism after localizing at $g$ (cf. [2], Proposition 2.8). Since $i_{F}^{*}\left(\beta_{\mathbb{R}^{n}}\right)=\beta_{F} \cdot\left[\Lambda_{\mathbb{C}}^{*}(N)\right]$ by the same argumentation with $A$ set equal to the identity, 
we get that $i_{F}^{*}\left(A_{*} \beta_{\mathbb{R}^{n}}\right)=\operatorname{sign} \operatorname{det}\left(A_{\mid F}\right) i_{F}^{*}\left(\beta_{\mathbb{R}^{n}}\right)$ and hence since $i_{F}^{*}$ is an isomorphism after localization at $g$, that $A_{*}\left(\beta_{\mathbb{R}^{n}}\right)=\operatorname{sign} \operatorname{det}\left(A_{\mid F}\right) \beta_{\mathbb{R}^{n}}$ after localization at $g$. Therefore, taking ind $\mathrm{t}_{\mathrm{t}}^{\Gamma}$ of both sides and using that $\operatorname{ind}_{\mathrm{t}}^{\Gamma}\left(\beta_{\mathbb{R}^{n}}\right)=1 \in \mathrm{R}(\Gamma)$, gives that $\operatorname{ind}_{\mathrm{t}}^{\Gamma}\left(A_{*} \beta_{\mathbb{R}^{n}}\right)=\operatorname{sign} \operatorname{det}\left(A_{\mid F}\right) 1 \in \mathrm{R}(\Gamma)_{g}$. Evaluation of characters at $g$ passes to the localization, and is compatible with evaluation before localization, whence evaluating the above expression at $g$ gives that $\operatorname{ind}_{\mathrm{t}}^{\Gamma}\left(A_{*} \beta_{\mathbb{R}^{n}}\right)(g)=\operatorname{sign} \operatorname{det}\left(A_{\mid F}\right)$ as required. This gives the second proof of Theorem 2.5.

\section{The Lefschetz theorem}

Let the countable group $G$ act isometrically, properly and co-compactly on the Riemannian manifold $X$ (it follows that $X$ is complete.) Let $\phi: X \rightarrow X$ be a smooth map. We are going to formulate and prove a Lefschetz fixed-point formula in this context using the discussion in Section 1 on Poincaré duality between $\Lambda=C_{0}(X) \rtimes G$ and $\hat{\Lambda}=C_{\tau}(X) \rtimes G$. To get an endomorphism of the algebra $\Lambda$ and to be adequate for the formulation of the Lefschetz theorem, we need a couple of assumptions on the map $\phi$ on the manifold $X$. First we require the following compatibility of the map $\phi$ and the $G$-action on $X$ :

Assumption 3.1. There is an automorphism $\zeta: G \rightarrow G$ such that

$$
\phi(\zeta(g) x)=g(\phi(x)) \quad \text { for all } x \in X .
$$

The assumption ensures that the maps $f \mapsto f \circ \phi$ and $g \mapsto \zeta(g)$ constitute a covariant pair for the action of $G$ on $C_{0}(X)$. We obtain an automorphism

$$
\alpha: C_{0}(X) \rtimes G \rightarrow C_{0}(X) \rtimes G .
$$

Next we require a transversality assumption on $\phi$. Suppose for the moment that the $G$-action on $X$ is free. Then $G \backslash X$ is a manifold, and since $\phi$ maps orbits to orbits, we obtain a smooth map $\dot{\phi}: G \backslash X \rightarrow G \backslash X$. In this case, we want to demand that $\dot{\phi}$ is in general position: that is, that its graph is transverse to the diagonal in $G \backslash X \times G \backslash X$.

By definition of the smooth structure on $G \backslash X$, this means the following: If $x \in X$, $g \in G$ such that $\phi(g x)=x$, then the map

$$
\mathrm{Id}-d(\phi \circ g)(x): T_{x} X \rightarrow T_{x} X
$$

is non-singular.

If the $G$-action is not free, $G \backslash X$ is not a manifold. But the reformulation of the condition that $\dot{\phi}$ be in general position given above still makes sense. We thus impose the following 
Assumption 3.2. For every $g \in G$, the smooth map $\phi \circ g: X \rightarrow X$ is in general position, i.e., (3.2) holds for all $x \in X$ with $\phi(g x)=x$.

The abstract Lefschetz theorem (see [9]) asserts that the Leftschetz number equals an index-theoretic pairing,i.e.,

$$
\operatorname{Lef}([\alpha])=\langle[\widehat{\alpha}], \Delta\rangle
$$

where $\operatorname{Lef}([\alpha])$ is the Lefschetz number of $\alpha$ defined by

$$
\operatorname{Lef}([\alpha]):=\operatorname{trace}_{s}\left(\alpha_{*}: \mathrm{K}_{*}\left(C_{0}(X) \rtimes G\right)_{\mathbb{Q}} \rightarrow \mathrm{K}_{*}\left(C_{0}(X) \rtimes G\right)_{\mathbb{Q}}\right)
$$

(which only depends on $[\alpha] \in \mathrm{KK}\left(C_{0}(X) \rtimes G, C_{0}(X) \rtimes G\right)$ ) and $[\hat{\alpha}]$ denotes the Poincaré dual of $[\alpha]$ which more exactly equals $\alpha_{*}\left(\sigma_{*} \hat{\Delta}\right) \in \mathrm{KK}(\mathbb{C}, \Lambda \hat{\otimes} \hat{\Lambda})$, where, as before, $\sigma: \hat{\Lambda} \hat{\otimes} \Lambda \rightarrow \Lambda \hat{\otimes} \hat{\Lambda}$ denotes the flip map.

Therefore, in order to prove Theorem 0.1 , we want to compute the pairing $\alpha_{*}\left(\sigma_{*} \hat{\Delta}\right) \hat{\otimes}_{\Lambda \hat{\otimes} \hat{\Lambda}} \Delta$, where $\alpha$ is as in (3.1). By functoriality, this is the same as $\sigma_{*} \hat{\Delta} \hat{\otimes}_{\Lambda \hat{\otimes} \hat{\Lambda}} \alpha^{*}(\Delta)$, which we will focus on instead.

We set

$$
F_{\varepsilon}:=\{(x, g) \in X \times G \mid \rho(\phi(g x), x)<\varepsilon\},
$$

where $\rho$ denotes the metric on $X$. Give $F_{\varepsilon}$ the structure of a $G$-space by restricting the following action of $G$ on $X \times G$ :

$$
h(x, g):=\left(h x, \zeta(h) g h^{-1}\right) .
$$

Let $F=F_{0}$ in the above notation, so $F=\{(x, g) \mid \phi(g x)=x\}$. Then $F_{\varepsilon}$ is a neighbourhood of $F$ and $F_{\varepsilon} \rightarrow F$ as $\varepsilon \rightarrow 0$. Note also that $G$ leaves $F_{\varepsilon}$ (and likewise $\left.F=F_{0}\right)$ invariant, as if $\rho(\phi(g x), x)<\varepsilon$ then

$$
\rho\left(\phi\left(\zeta(h) g h^{-1} h x\right), h x\right)=\rho(h \phi(g x), h x)=\rho(\phi(g x), x)<\varepsilon .
$$

Let $V_{\varepsilon}$ be the set of first coordinates of points in $F_{\varepsilon}$. Then $V_{\varepsilon}$ is a $G$-set for $\varepsilon \geq 0$. Let $V:=V_{0}$.

Lemma 3.3. The set $V$ is discrete. Furthermore, if $\delta>0$, there exists $\varepsilon>0$ such that every component of $V_{\varepsilon}$ is contained in a $\delta$-ball in $X$ with center in $V$.

Proof. Suppose $\left(x_{j}\right)$ and $\left(g_{j}\right)$ are sequences in $X$ and $G$ respectively such that $\phi\left(g_{j} x_{j}\right)=x_{j}$, the $x_{j}$ are all distinct, and $x_{j} \rightarrow x_{0}$ for some $x_{0}$. Let $h_{j}$ such that $\zeta\left(h_{j}\right)=g_{j}^{-1}$. Then $\phi\left(x_{j}\right)=h_{j} x_{j}$. Since $x_{j} \rightarrow x_{0}, \phi\left(x_{j}\right) \rightarrow \phi\left(x_{0}\right)$, and hence $h_{j} x_{j} \rightarrow \phi\left(x_{0}\right)$. But then

$$
\rho\left(h_{j} x_{0}, \phi\left(x_{0}\right)\right) \leq \rho\left(h_{j} x_{0}, h_{j} x_{j}\right)+\rho\left(h_{j} x_{j}, \phi\left(x_{0}\right)\right) \rightarrow 0 .
$$

But since the $G$-action is proper, there are only finitely many $h \in G$ which map $x_{0}$ to any fixed, pre-compact neighbourhood of $\phi\left(x_{0}\right)$. Hence $h_{j}=h$ for some $h$ and 
almost all $j$. We may assume $h_{j}=h$ for all $j$, which gives that $g_{j}=g$ for all $j$ and then $\phi \circ g$ has an accumulation point amongst its fixed points, which contradicts Assumption 3.2. This argument proves that $V$ is discrete.

For the second statement, observe that $\left\{V_{1 / n}\right\}$ is a nested sequence whose intersection is $V$. Using the $G$-compactness of $X$, we see that, given $\delta>0$, there exists $n \in \mathbb{N}$ such that $V_{1 / n}$ is contained in the $\delta$-neighbourhood of $V$. By the first statement, the second statement now follows.

Since $V$ is discrete and the $G$-action on $X$ is co-compact, $V$ splits into finitely many $G$-orbits. Observe that the set of such orbits has an obvious correspondence with the set

$$
\operatorname{Fix}(\dot{\phi}):=\{p \in X \mid \dot{\phi}(\dot{p})=\dot{p}\},
$$

where $\dot{\phi}$ is the induced map $G \backslash X \rightarrow G \backslash X$ and $\dot{p}$ denotes an orbit of $p$. Let us denote each $G$-orbit in $V$ corresponding to each point $p \in \operatorname{Fix}(\dot{\phi})$ by $V_{p}$.

The $G$-set $F$ admits a similar decomposition, $F=\bigsqcup F_{p}$, where $F_{p}=\left\{(x, h) \in F \mid x \in V_{p}\right\}$. For each $V_{p}$ fix an element $g_{p} \in G$ such that $\phi\left(g_{p} p\right)=p$. Let $L_{p}:=g_{p} K_{p}$ be the coset of $K_{p}:=\operatorname{Stab}_{G}(p)$. Then one can see that $L_{p}=\{g \in G \mid \phi(g p)=p\}$.

From this, we get the following. Consider a point $g p \in V_{p}$. Then there exists $h \in G$ such that $\phi(h g p)=g p$, and hence $\phi\left(\zeta(g)^{-1} h g p\right)=p=\phi\left(g_{p} p\right)$, so that $g_{p} p=\zeta(g)^{-1} h g p$ and $g_{p}^{-1} \zeta(g)^{-1} h g \in K_{p}$. Hence $h$ lies in the twisted conjugate $\zeta(g) L_{p} g^{-1}$ of $L_{p}$. The converse of this statement is also true.

Hence we can write

$$
V_{p}=\left\{g p \mid g K_{p} \in G / K_{p}\right\}, \quad F_{p}=\left\{(g p, h) \mid g K_{p} \in G / K_{p}, h \in \zeta(g) L_{p} g^{-1}\right\} .
$$

Similarly, we get decompositions of $V_{\varepsilon}$ and $F_{\varepsilon}$. By Lemma 3.3, we may choose $\delta>0$ small enough so that all the $\delta$-balls centered at the points of $V$ are disjoint and therefore there exists $\varepsilon>0$ such that

$$
V_{\varepsilon}=\bigsqcup_{\substack{p \in \operatorname{ix}(\dot{\phi}) \\ g K p \in G / K_{p}}} V_{\varepsilon, g p},
$$

where $V_{\varepsilon, g p}$ is the part of $V_{\varepsilon}$ which is contained in the $\delta$-ball centered at the point $g p \in V$. Similarly,

$$
F_{\varepsilon}=\underset{\substack{p \in \operatorname{cic}(\dot{\phi}) \\ g K_{p} \in G / K_{p}}}{ } F_{\varepsilon, g p},
$$

where

$$
F_{\varepsilon, g p}=\left\{(x, h) \in X \times G \mid x \in V_{\varepsilon, g p}, h \in \zeta(g) L_{p} g^{-1}\right\} .
$$

In what follows, we shall describe the pairing $\sigma_{*} \hat{\Delta} \hat{\otimes}_{\Lambda} \hat{\otimes} \hat{\Lambda} \alpha^{*} \Delta$ as a direct sum of Kasparov products which live on the Hilbert spaces $L^{2}\left(\Lambda_{\mathbb{C}}^{*}\left(V_{\varepsilon, p}\right)\right)^{\Gamma_{p, g}}$, where $\Gamma_{p, g} \subseteq K_{p}$ denotes the stabilizer of $g \in L_{p}$ under the conjugation action 
$g \mapsto \zeta(h) g h^{-1}$. These summands can then be computed via the results of the previous section. We start with a careful description of the Hilbert space (recall Definitions 1.10 and 1.11). It is the tensor product of the right Hilbert $\Lambda \hat{\otimes} \hat{\Lambda}$-module $\varepsilon$ described prior to Definition 1.11, and the Hilbert space $L^{2}\left(\Lambda_{\mathbb{C}}^{*} X\right) \hat{\otimes} \ell^{2} G$ occurring in connection with the fundamental class $\Delta$, twisted by the automorphism $\alpha$ induced from $\phi$ and $\zeta$ (see (3.1)). Here and throughout we write $\Lambda$ for $C_{0}(X) \rtimes G$ and $\hat{\Lambda}$ for $C_{\tau}(X) \rtimes G$.

After twisting $\Delta$ by $\alpha$, we obtain the Hilbert space $L^{2}\left(\Lambda_{\mathbb{C}}^{*} X\right) \hat{\otimes} \ell^{2} G$ equipped with a twisted representation of $\Lambda \hat{\otimes} \hat{\Lambda}$ whose explicit form we state for the record (compare with the untwisted version in (1.6) and (1.7)): the algebra $\Lambda=C_{0}(X) \rtimes G$ acts via the covariant pair

$$
f\left(\xi \hat{\otimes} e_{g}\right)=g^{-1}(f \circ \phi) \xi \hat{\otimes} e_{g}, \quad h \cdot\left(\xi \hat{\otimes} e_{g}\right)=\xi \hat{\otimes} e_{\zeta}(h) g
$$

for $f \in C_{0}(X), h \in G$ and $\xi \hat{\otimes} e_{g} \in L^{2}\left(\Lambda_{\mathbb{C}}^{*} X\right) \hat{\otimes} \ell^{2} G$. The algebra $\hat{\Lambda}=C_{\tau}(X) \rtimes G$ acts by the covariant pair

$$
\varphi\left(\xi \hat{\otimes} e_{g}\right)=\varphi \xi \hat{\otimes} e_{g}, \quad h \cdot\left(\xi \hat{\otimes} e_{g}\right)=h(\xi) \hat{\otimes} e_{g h^{-1}}
$$

for $\varphi \in C_{\tau}(X), h \in G$ and $\xi \hat{\otimes} e_{g} \in L^{2}\left(\Lambda_{\mathbb{C}}^{*} X\right) \hat{\otimes} \ell^{2} G$. Recall that $\varepsilon$ is the completion of $C_{c}(X) \mathcal{F}_{U} \widehat{\otimes} \mathbb{C} G$ with respect to a certain inner product, where $U=$ $\{(x, y) \mid \rho(x, y)<\varepsilon\}$ for some $\varepsilon>0$ from Remark 1.5 (iv). We may choose (and fix) $\varepsilon$ such that (3.4) is satisfied for a suitable $\delta>0$.

Notice that there is a well defined inclusion of the algebraic tensor product $C_{c}(X) \mathcal{F}_{U} \odot \mathbb{C} G$ into $\Lambda \hat{\otimes} \hat{\Lambda}$ given by sending the elementary tensor $\alpha \hat{\otimes}[h]$ to the element $\left.\alpha([h] \hat{\otimes}[h]) \in\left(C_{0}(X) \hat{\otimes} C_{\tau}(X)\right) \rtimes(G \times G)\right) \cong \Lambda \hat{\otimes} \hat{\Lambda}$. Using this inclusion, we obtain a natural pairing

$$
M:\left(C_{c}(X) \mathscr{F}_{U} \odot \mathbb{C} G\right) \times\left(L^{2}\left(\Lambda_{\mathbb{C}}^{*} X\right) \hat{\otimes} \ell^{2} G\right) \rightarrow L^{2}\left(\Lambda_{\mathbb{C}}^{*} X\right) \hat{\otimes} \ell^{2} G
$$

given by applying the action of $\Lambda \hat{\otimes} \hat{\Lambda}$ as described in (3.7) to the image of $C_{c}(X) \mathcal{F}_{U} \odot \mathbb{C} G$ under the above described inclusion.

Recall that $F_{\varepsilon}=\{(x, g) \in X \times G \mid \rho(\phi(g x), x)<\varepsilon\}$. We denote by $L^{2}\left(\Lambda_{\mathbb{C}}^{*} F_{\varepsilon}\right)$ the set of all $\xi \in L^{2}\left(\Lambda_{\mathbb{C}}^{*} X\right) \hat{\otimes} \ell^{2} G$ which live on $F_{\varepsilon}$ in the obvious sense (by viewing the elements of $L^{2}\left(\Lambda_{\mathbb{C}}^{*} X\right) \hat{\otimes} \ell^{2} G$ as sections on $\left.X \times G\right)$. We then get

Lemma 3.4. Let $\alpha \hat{\otimes}[h] \in C_{c}(X) \mathcal{F}_{U} \odot \mathbb{C} G$ act on $L^{2}\left(\Lambda_{\mathbb{C}}^{*} X\right) \hat{\otimes} \ell^{2} G$ as described above. Then $(\alpha \hat{\otimes}[h]) \cdot L^{2}\left(\Lambda_{\mathbb{C}}^{*} X\right) \hat{\otimes} \ell^{2} G \subseteq L_{c}^{2}\left(\Lambda_{\mathbb{C}}^{*} F_{\varepsilon}\right)$, where $L_{c}^{2}\left(\Lambda_{\mathbb{C}}^{*} F_{\varepsilon}\right)$ denotes the set of $L^{2}$-sections on $F_{\varepsilon}$ which vanish outside some compact subset of $F_{\varepsilon}$.

Proof. If we regard the elements of $L^{2}\left(\Lambda_{\mathbb{C}}^{*} X\right) \hat{\otimes} \ell^{2} G$ as sections on $X \times G$ in the canonical way, it follows from (3.7) that the action of $\alpha \hat{\otimes}[h]$ on such section $\mu \in L^{2}\left(\Lambda_{\mathbb{C}}^{*} X\right) \hat{\otimes} \ell^{2} G$ is given by the formula

$$
\left.((\alpha \hat{\otimes}[h]) \cdot \mu)(x, g)=\alpha(\phi(g x), x) d_{h}^{x}\left(\mu\left(h^{-1} x, \zeta\left(h^{-1}\right) g h\right)\right)\right),
$$


where $d_{h}^{x}: \operatorname{Cl}\left(T_{h^{-1} x_{x}} X\right) \rightarrow \operatorname{Cl}\left(T_{x} X\right)$ is the isomorphism underlying the action of $G$ on $C_{\tau}(X)$. Thus the result follows directly from the fact that $\alpha$ is compactly supported in $U_{\varepsilon}=\{(x, y) \mid \rho(x, y)<\varepsilon\}$.

In what follows let $P \subseteq X$ be a fixed set of representatives for $\operatorname{Fix}(\dot{\phi})=G \backslash V$, with $V$ as in the discussion in the beginning of this section. Let

$$
S:=\left\{\left(p, g K_{p}\right) \mid p \in P, g K_{p} \in G / K_{p}\right\},
$$

where $K_{p}$ denotes the stabilizer of $p$ in $G$. Recall from (3.5) that for $\varepsilon>0$ small enough, the set $F_{\varepsilon}$ decomposes into a disjoint union

$$
F_{\varepsilon}=\underset{\left(p, g K_{p}\right) \in S}{\bigsqcup} F_{\varepsilon, g p},
$$

with

$$
F_{\varepsilon, g p}=\left\{(x, h) \in X \times G \mid x \in V_{\varepsilon, g p}, h \in \zeta(g) L_{p} g^{-1}\right\} .
$$

Note that $G$ acts unitarily on $L^{2}\left(\Lambda_{\mathbb{C}}^{*} F_{\varepsilon}\right)$ via

$$
(s \mu)(x, g)=d_{s}^{x}\left(\mu\left(s^{-1} x, \zeta\left(s^{-1}\right) g s\right)\right)
$$

for $s \in G, \mu \in L_{c}^{2}\left(\Lambda_{\mathbb{C}}^{*} F_{\varepsilon}\right)$, where, by abuse of notation, $d_{s}^{x}: \Lambda_{\mathbb{C}}^{*}\left(T_{s^{-1} x} X\right) \rightarrow$ $\Lambda_{\mathbb{C}}^{*}\left(T_{x} X\right)$ is the isometry induced by the differential $d_{s}^{x}: T_{s^{-1} x} X \rightarrow T_{x} X$. This action restricts to well-defined actions of $K_{p}=\operatorname{Stab}_{G}(p)$ on $F_{\varepsilon, p}$ for all $p \in P$. As usual, we let $L^{2}\left(\Lambda_{\mathbb{C}}^{*} F_{\varepsilon, p}\right)^{K_{p}}$ denote the $K_{p}$-invariant elements in $L^{2}\left(\Lambda_{\mathbb{C}}^{*} F_{\varepsilon, p}\right)$.

In what follows, we equip $L_{c}^{2}\left(\Lambda_{\mathbb{C}}^{*} F_{\varepsilon}\right)$ with a new inner product given by

$$
\langle\mu, \nu\rangle=\sum_{s \in G}\langle s(\mu), \nu\rangle_{L^{2}\left(\Lambda_{\mathbb{C}}^{*} F_{\varepsilon}\right)}
$$

with action of $G$ on $L_{c}^{2}\left(\Lambda_{\mathbb{C}}^{*} F_{\varepsilon}\right)$ as explained above. Note that this inner product makes sense, since $G$ acts properly on $F_{\varepsilon}$ and $\mu$ and $v$ are compactly supported. We denote by $\mathscr{H}_{\varepsilon}$ the Hausdorff completion of $L_{c}^{2}\left(\Lambda_{\mathbb{C}}^{*} F_{\varepsilon}\right)$ with respect to this inner product.

Lemma 3.5. Consider the composition $\Phi=\Psi \circ M$ of maps

$$
\mathcal{E} \hat{\otimes}_{\Lambda \hat{\otimes} \hat{\Lambda}}\left(L^{2}\left(\Lambda_{\mathbb{C}}^{*} X\right) \hat{\otimes} \ell^{2} G\right) \stackrel{M}{\rightarrow} \mathscr{H}_{\varepsilon} \stackrel{\Psi}{\rightarrow} \bigoplus_{p \in P} L^{2}\left(\Lambda_{\mathbb{C}}^{*} F_{\varepsilon, p}\right) K_{p},
$$

where $M$ is given on elementary tensors by the pairing of Lemma 3.4 and where

$$
\Psi(\eta)=\left.\bigoplus_{p \in P} \frac{1}{\sqrt{\left|K_{p}\right|}} \sum_{s \in G} s \eta\right|_{F_{\varepsilon, p}}
$$

for $\eta \in L_{c}^{2}\left(\Lambda_{\mathbb{C}}^{*} F_{\varepsilon}\right)$. Then $\Phi$ is an isometric isomorphism of Hilbert spaces. 
Proof. Using formulas (1.8), (3.6), (3.7) and (3.8), we compute for all $\alpha_{i} \widehat{\otimes}\left[h_{i}\right] \in$ $C_{c}(X) \tilde{F}_{U} \widehat{\otimes} \mathbb{C} G$ and $\mu_{i} \in L^{2}\left(\Lambda_{\mathbb{C}}^{*} X\right) \hat{\otimes} \ell^{2} G, i=1,2$ :

$$
\begin{aligned}
& \left\langle\left(\alpha_{1} \hat{\otimes}\left[h_{1}\right]\right) \hat{\otimes}_{\Lambda \hat{\otimes} \hat{\Lambda}} \mu_{1},\left(\alpha_{2} \hat{\otimes}\left[h_{2}\right]\right) \hat{\otimes}_{\Lambda \hat{\otimes} \hat{\Lambda}} \mu_{2}\right\rangle_{\mathcal{E} \hat{\otimes}} \hat{\Lambda} \hat{\Lambda} L^{2}\left(\Lambda_{\mathbb{C}}^{*} X\right) \hat{\otimes} \ell^{2} G \\
& =\sum_{g \in G} \int_{X}\left\langle\left(\left\langle\alpha_{2} \hat{\otimes}\left[h_{2}\right], \alpha_{1} \hat{\otimes}\left[h_{1}\right]\right\rangle_{\mathcal{E}} \cdot \mu_{1}\right)(x, g), \mu_{2}(x, g)\right\rangle d x \\
& =\sum_{g \in G} \sum_{s \in G} \int_{X}\left\langle\left(h_{2}^{-1}\left(\alpha_{2}^{*} s\left(\alpha_{1}\right)\right)\left[h_{2}^{-1} s h_{1}\right] \hat{\otimes}\left[h_{2}^{-1} s\right]\right) \cdot \mu_{1}(x, g), \mu_{2}(x, g)\right\rangle d x \\
& =\sum_{g \in G} \sum_{s \in G} \int_{X}\left\langle h_{2}^{-1}\left(\alpha_{2}^{*} s\left(\alpha_{1}\right)\right)(\phi(g x), x) d_{h_{2}^{-1} s h_{1}}^{x}\right. \\
& \left.\quad\left(\mu_{1}\left(h_{1}^{-1} s^{-1} h_{2} x, \zeta\left(s^{-1} h_{2}\right) g h_{2}^{-1} s h_{1}\right)\right), \mu_{2}(x, g)\right\rangle d x \\
& =\sum_{g \in G} \sum_{s \in G} \int_{X}\left\langle d_{h_{2}^{-1}}^{x}\left(\alpha_{2}^{*} s\left(\alpha_{1}\right)\left(h_{2} \phi(g x), h_{2} x\right)\right) d_{h_{2}^{-1} s h_{1}}^{x}\right. \\
& \left.\quad\left(\mu_{1}\left(h_{1}^{-1} s^{-1} h_{2} x, \zeta\left(s^{-1} h_{2}\right) g h_{2}^{-1} s h_{1}\right)\right), \mu_{2}(x, g)\right\rangle d x .
\end{aligned}
$$

Now, applying on both sides the unitary transformation $v \mapsto h_{2} v$ given by the formula in (3.9), the above term transforms into

$$
\begin{aligned}
& =\sum_{g \in G} \sum_{s \in G} \int_{X}\left\langle\left(\alpha_{2}^{*} s\left(\alpha_{1}\right)\right)(\phi(g x), x) d_{s h_{1}}^{x}\left(\mu_{1}\left(h_{1}^{-1} s^{-1} x, \zeta\left(s^{-1}\right) g s h_{1}\right)\right),\right. \\
& \left.=d_{h_{2}}^{x}\left(\mu_{2}\left(h_{2}^{-1} x, \zeta\left(h_{2}^{-1}\right) g h_{2}\right)\right)\right\rangle d x \\
& =\sum_{s \in G} \int_{X}\left\langle\left( d_{s}^{x}\left(\alpha_{1}\right)\left(s^{-1} \phi(g x), s^{-1} x\right) d_{s h_{1}}^{x}\left(\mu_{1}\left(h_{1}^{-1} s^{-1} x, \zeta\left(s^{-1}\right) g s h_{1}\right)\right),\right.\right. \\
& \left.\alpha_{2}(\phi(g x), x) d_{h_{2}}^{x}\left(\mu_{2}\left(h_{2}^{-1} x, \zeta\left(h_{2}^{-1}\right) g h_{2}\right)\right)\right\rangle d x \\
& =\sum_{s \in G} \sum_{g \in G} \int_{X}\left\langle s\left(\left(\alpha_{1} \hat{\otimes}\left[h_{1}\right]\right) \cdot \mu_{1}\right)(x, g),\left(\alpha_{2} \hat{\otimes}\left[h_{2}\right]\right) \cdot \mu_{2}(x, g)\right\rangle d x \\
& =\sum_{s \in G}\left\langle s\left(\left(\alpha_{1} \hat{\otimes}\left[h_{1}\right]\right) \cdot \mu_{1}\right),\left(\alpha_{2} \hat{\otimes}\left[h_{2}\right]\right) \cdot \mu_{2}\right\rangle_{L^{2}\left(\Lambda_{\mathbb{C}}^{*} F_{\varepsilon}\right)} .
\end{aligned}
$$

This demonstrates that $M$ extends to a well-defined unitary homomorphism from $\mathcal{E} \hat{\otimes}_{\Lambda \hat{\otimes} \hat{\Lambda}}\left(L^{2}\left(\Lambda_{\mathbb{C}}^{*} X\right) \hat{\otimes} \ell^{2} G\right)$ to $\mathscr{H}_{\varepsilon}$, and it is not difficult to see that it has dense image. Thus the result will follow if we can show that $\Psi: \mathscr{H}_{\varepsilon} \rightarrow \bigoplus_{p \in P} L^{2}\left(\Lambda_{\mathbb{C}}^{*} F_{\varepsilon, p}\right)^{K_{p}}$ is also isometric (it clearly has dense image). After decomposing $F_{\varepsilon}$ into the disjoint union $\bigsqcup_{\left(p, g K_{p}\right) \in S} F_{\varepsilon, g p}$, we may assume without loss of generality that $P=\{p\}$ is a single point. Then $L_{c}^{2}\left(\Lambda_{\mathbb{C}}^{*} F_{\varepsilon}\right)$ can be written as the set of finite sums $\eta=\sum_{g K_{p}} \eta_{g}$ with $\eta_{g}$ supported on $F_{\varepsilon, g p}$. Each such function is of the form $g \eta^{\prime}$ for some $\eta^{\prime} \in L^{2}\left(\Lambda_{\mathbb{C}}^{*} F_{\varepsilon, p}\right)$. So assume now that $\eta, v \in L^{2}\left(\Lambda_{\mathbb{C}}^{*} F_{\varepsilon, p}\right)$ and $g, h \in G$. Then

$$
\begin{aligned}
\langle g \eta, h v\rangle_{\mathscr{H}_{\varepsilon}} & =\sum_{s \in G}\langle s g \eta, h v\rangle_{L^{2}\left(\Lambda_{\mathbb{C}}^{*} F_{\varepsilon}\right)} \\
& \stackrel{h}{=} \sum_{s \in G}\langle s \eta, v\rangle_{L^{2}\left(\Lambda_{\mathbb{C}}^{*} F_{\varepsilon}\right)}=\sum_{s \in K_{p}}\langle s \eta, v\rangle_{L^{2}\left(\Lambda_{\mathbb{C}}^{*} F_{\varepsilon}\right)} .
\end{aligned}
$$


On the other hand, we have

$$
\Psi(g \eta)=\left.\frac{1}{\sqrt{\left|K_{p}\right|}} \sum_{s \in G} s g \eta\right|_{F_{\varepsilon, p}}=\left.\frac{1}{\sqrt{\left|K_{p}\right|}} \sum_{s \in K_{p}} s \eta\right|_{F_{\varepsilon, p}}
$$

from which we get

$$
\begin{aligned}
\langle\Psi(g \eta), \Psi(h v)\rangle_{L^{2}\left(\Lambda_{C}^{*} F_{\varepsilon, p}\right)} & =\frac{1}{\left|K_{p}\right|} \sum_{s, t \in K_{p}}\langle s \eta, t v\rangle_{L^{2}\left(\Lambda_{C}^{*} F_{\varepsilon, p}\right)} \\
& =\sum_{s \in K_{p}}\langle s \eta, v\rangle_{L^{2}\left(\Lambda_{C}^{*} F_{\varepsilon, p}\right)},
\end{aligned}
$$

which now proves that $\langle\Psi(\eta), \Psi(v)\rangle_{L^{2}\left(\Lambda_{C}^{*} F_{\varepsilon, p}\right)}=\langle\eta, v\rangle_{\mathscr{H}_{\varepsilon}}$ for all $\eta, v \in L_{c}^{2}\left(\Lambda_{\mathbb{C}}^{*} F_{\varepsilon}\right)$.

Since $K_{p}$ acts on $F_{\varepsilon, p}=V_{\varepsilon, p} \times L_{p}$ by $h \cdot(x, g)=\left(h x, \zeta(h) g h^{-1}\right)$ as defined in (3.3), and also since one can consider $L^{2}\left(\Lambda_{\mathbb{C}}^{*} F_{\varepsilon, p}\right)$ as a direct sum of copies of $L^{2}\left(\Lambda_{\mathbb{C}}^{*} V_{\varepsilon, p}\right)$, one summand for each point in $L_{p}$, we have

$$
L^{2}\left(\Lambda_{\mathbb{C}}^{*} F_{\varepsilon, p}\right)^{K_{p}}=\bigoplus_{g} L^{2}\left(\Lambda_{\mathbb{C}}^{*} V_{\varepsilon, p}\right)^{\Gamma_{p, g}}
$$

where $g$ runs through a given set $\Sigma_{p}$ of representatives for the orbits in $L_{p}$ under the twisted conjugation by $K_{p}$ and $\Gamma_{p, g} \subset K_{p}$ denotes the stabilizer of $g$ under this action. Thus, combining this observation with the above lemma we get

$$
\mathcal{E} \hat{\otimes}_{\Lambda \hat{\otimes} \hat{\Lambda}}\left(L^{2}\left(\Lambda_{\mathbb{C}}^{*} X\right) \hat{\otimes} \ell^{2} G\right) \cong \bigoplus_{\Sigma} L^{2}\left(\Lambda_{\mathbb{C}}^{*} V_{\varepsilon, p}\right)^{\Gamma_{p, g}}=\bigoplus_{\Sigma} \mathscr{H}_{p, g}^{\Gamma_{p, g}},
$$

with $\Sigma=\cup_{p \in P} \Sigma_{p}$ and $\mathscr{H}_{p, g}=L^{2}\left(\Lambda_{\mathbb{C}}^{*} V_{\varepsilon, p}\right)$.

We are now going to compute the operator. To this end let $g \in L_{p}$. Since $\rho(\phi(g x), x)<\varepsilon$ for all $x \in V_{\varepsilon, p}$ and $g \in L_{p}$, we have $(\phi(g x), x) \in U_{\varepsilon}$ for all such $x$ and $g$. Thus we have a well-defined vector field $\theta_{p, g}: V_{\varepsilon, p} \rightarrow T V_{\varepsilon, p}$ given by $\theta_{p, g}(x)=\theta_{\varepsilon}(\phi(g x), x)$ with $\theta_{\varepsilon}(z, x)=\frac{\rho(z, x)}{\varepsilon} d_{x} \rho(z, x)$ as in Remark 1.5 (iv). It determines a class $\Theta_{g, p} \in \mathrm{KK}^{\Gamma_{g, p}}\left(\mathbb{C}, C_{\tau}\left(V_{\varepsilon, p}\right)\right)$ as in the previous section. Indeed, since $\left\|d_{x} \rho(z, x)\right\|=1$ for all $z, x \in X$ with $z \neq x$, it follows that $\theta_{p, g}(x)^{2}-1=$ $\left\|\theta_{p, g}(x)\right\|^{2}-1 \rightarrow 0$ if $x \rightarrow \infty$ in $V_{\varepsilon, p}$, and therefore the class $\Theta_{p, g}$ is given directly via Clifford multiplication of $\theta_{p, g}$ on $C_{\tau}\left(V_{\varepsilon, p}\right)$. On the other hand, we can consider the Dirac-class $\left[D_{p, g}\right]=\left[D_{V_{\varepsilon, p}}\right] \in \mathrm{KK}^{\Gamma_{p, g}}\left(C_{\tau}\left(V_{\varepsilon, p}\right), \mathbb{C}\right)$. It is represented by the restriction $F_{p, g}$ of the bounded de Rham operator $F=D\left(1+D^{2}\right)^{-1 / 2}$ to $L^{2}\left(\Lambda_{\mathbb{C}}^{*}\left(V_{\varepsilon, p}\right)\right)$.

The Kasparov product $\Theta_{p, g} \otimes_{C_{\tau}\left(V_{p, \varepsilon}\right)}\left[D_{p, g}\right] \in \mathrm{KK}^{\Gamma_{p, g}}(\mathbb{C}, \mathbb{C})$ is represented by the pair $\left(\mathscr{H}_{p, g}, P_{p, g}\right)$, with $\mathscr{H}_{p, g}=L^{2}\left(\Lambda_{\mathbb{C}}^{*}\left(V_{p, \varepsilon}\right)\right)$ and

$$
P_{p, g}=\lambda_{\theta_{p, g}(x)}+\lambda_{\theta_{p, g}(x)}^{*}+\sqrt{1-\left\|\theta_{p, g}(x)\right\|^{2}} F_{p, g}
$$


(Compare with (2.1) in the proof of Lemma 2.2 above.) Since $P_{p, g}$ is $\Gamma_{p, g}$-invariant, it restricts to an operator $Q_{p, g}$ on the subspace of the $\Gamma_{p, g}$-invariant vectors in $\mathscr{H}_{p, g}$. We then get well-defined classes

$$
\left[\left(\mathcal{H}_{p, g}^{\Gamma_{p, g}}, Q_{p, g}\right)\right] \in \mathrm{KK}(\mathbb{C}, \mathbb{C})
$$

for each pair $(p, g) \in S$. We now get

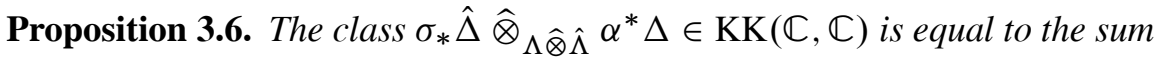

$$
\sum_{(p, g) \in \Sigma}\left[\left(H_{p, g}^{\Gamma_{p, g}}, Q_{p, g}\right] \in \mathrm{KK}(\mathbb{C}, \mathbb{C}) .\right.
$$

Proof. Recall the operators $F \hat{\otimes} 1=D\left(1+D^{2}\right)^{-1 / 2} \hat{\otimes} 1$ and $\theta_{G}$ from Definitions 1.10 and 1.11. Under the identification, $\mathcal{E} \hat{\otimes}_{\Lambda \hat{\otimes} \hat{\Lambda}} L^{2}\left(\Lambda_{\mathbb{C}}^{*} X\right) \hat{\otimes} \ell^{2} G \cong$ $\bigoplus_{p \in P} L^{2}\left(\Lambda_{\mathbb{C}}^{*} F_{\varepsilon, p}\right)^{K_{p}}$ of Lemma 3.5, $\theta_{G} \hat{\otimes} 1$ on $\varepsilon \hat{\otimes}_{\Lambda \hat{\otimes} \hat{\Lambda}} L^{2}\left(\Lambda_{\mathbb{C}}^{*} X\right) \hat{\otimes} \ell^{2} G$ corresponds to the operator $\tilde{\Theta}$ given point-wise by $\lambda_{\tilde{\theta}(x, g)}+\lambda_{\tilde{\theta}(x, g)}^{*}$, with $\tilde{\theta}(x, g)=$ $\theta(\phi(g x), x)$ and where $\lambda_{v}$ denotes exterior multiplication with $v$. Observe that

$$
\begin{aligned}
h \tilde{\theta}(x, g) & =d_{h}^{x}\left(\tilde{\theta}\left(h^{-1} x, \zeta(h)^{-1} g h\right)\right) \\
& =d_{h}^{x}\left(\theta\left(h^{-1} \phi(g x), h^{-1} x\right)\right) \\
& =\theta(\phi(g x), x)=\tilde{\theta}(x, g),
\end{aligned}
$$

since $\theta$ is $G$-invariant. Thus $\tilde{\Theta}$ descends to an operator on each $L^{2}\left(\Lambda_{\mathbb{C}}^{*} F_{\varepsilon, p}\right)^{K_{p}}$. Under the decomposition $L^{2}\left(\Lambda_{\mathbb{C}}^{*} F_{\varepsilon, p}\right)^{K_{p}} \cong \bigoplus_{g \in \Sigma_{p}} \mathscr{H}_{p, g}^{\Gamma_{p, g}}$ this operator becomes the sum $\bigoplus_{g \in \Sigma_{p}}\left(\lambda_{\theta_{p, g}(x)}+\lambda_{\theta_{p, g}(x)}^{*}\right)$ as in (3.10). Similarly, the operator $1 \hat{\otimes}(F \hat{\otimes} 1)$ descents to the sum of the de Rham operators $F_{p, g}$ under the decomposition $\mathcal{E} \hat{\otimes}_{\Lambda \hat{\otimes}} \hat{\Lambda}$ $L^{2}\left(\Lambda_{\mathbb{C}}^{*} X\right) \hat{\otimes} \ell^{2} G \cong \bigoplus_{(p, g) \in \Sigma} \mathscr{H}_{p, g}^{\Gamma_{p, g}}$. To check that the sum of the operators

$$
Q_{p, q}=\lambda_{\theta_{p, g}(x)}+\lambda_{\theta_{p, g}^{*}(x)}^{*}+\sqrt{1-\left\|\theta_{p, g}(x)\right\|^{2}} F_{p, g}
$$

on $\bigoplus_{(p, g) \in \Sigma} \mathscr{H}_{p, g}^{\Gamma_{p, g}}$ satisfies the axioms of a Kasparov product as explained in Remark 2.3, it is enough to check that $T:=\bigoplus_{(p, g)} F_{p, g}$ is a $F \hat{\otimes} 1$ connection. But this follows from the description of the isomorphism $\Phi$ of Lemma 3.5: If $\xi=\alpha \hat{\otimes}[h] \in C_{c}(X) \mathscr{F}_{U} \hat{\otimes} \mathbb{C} G$, and if we consider $T$ as an operator on $\bigoplus_{p \in P} L^{2}\left(\Lambda_{\mathbb{C}}^{*} F_{\varepsilon, p}\right)^{K_{p}}$ via the obvious identifications, then the operator

$$
F_{\xi}:=\Theta_{\xi}(F \hat{\otimes} 1)-(-1)^{\operatorname{deg} \xi \operatorname{deg} F} T \Theta_{\xi} \in \mathbb{B}\left(L^{2}\left(\Lambda_{\mathbb{C}}^{*}(X) \hat{\otimes} l^{2} G, \bigoplus_{p \in P} L^{2}\left(\Lambda_{\mathbb{C}}^{*} F_{\varepsilon, p}\right)^{K_{p}}\right)\right.
$$

can be described as the composition of the operator $\left[\Pi(\alpha[h] \hat{\otimes}[h]), F_{2} \hat{\otimes} 1\right] \epsilon$ $\mathbb{K}\left(L^{2}\left(\Lambda_{\mathbb{C}}^{*}(X) \hat{\otimes} l^{2} G\right)\right.$ followed by a projection to the $L^{2}$-sections on a finite union of 
components in $F_{\varepsilon}$ (which are determined by the support of $\alpha$ ), and then followed by the operator $\Psi$ of Lemma 3.5, which becomes bounded when restricted to the set of $L^{2}$-sections on a fixed finite number of components of $F_{\varepsilon}$. Thus $F_{\xi}$ is a composition of a compact operator by bounded operators, hence it is compact. This finishes the proof.

Finally, in order to get the corresponding integer for the class $\hat{\Delta} \hat{\otimes}_{\Lambda} \hat{\otimes} \hat{\Lambda} \alpha^{*} \Delta \in$ $\mathrm{KK}(\mathbb{C}, \mathbb{C}) \cong \mathbb{Z}$, we want to compute the index of the operator $H$, which is the sum of indexes of $Q_{p, g}$ for all $(p, g) \in \Sigma$. That is,

$$
\sigma_{*} \hat{\Delta} \hat{\otimes}_{\Lambda \hat{\otimes} \hat{\Lambda}} \alpha^{*} \Delta=\sum_{(p, g) \in \Sigma} \operatorname{ind}\left(Q_{p, g}\right) .
$$

We do this by first computing the classes $\left[\left(\mathscr{H}_{p, g}, P_{p, g}\right)\right] \in \mathrm{R}\left(\Gamma_{p, g}\right)$ and then computing from this the index of $Q_{p, g}$ as in Corollary 2.7.

To compute $\left[\left(\mathscr{H}_{p, g}, P_{p, g}\right)\right] \in \mathrm{R}\left(\Gamma_{p, g}\right)$, we linearize using the exponential map so that we are considering a similar problem in Euclidean space. So in what follow we may assume that $p=0$ is the origin in $\mathbb{R}^{n}$ and $V_{\varepsilon, p}$ is some open neighborhood of $p=0$ in $\mathbb{R}^{n}$. By choosing $\varepsilon$ small enough, we may further assume that $p=0$ is the only fixed point of the differential map $x \mapsto \phi(g x)$. This implies that the vector field $\theta_{p, g}: V_{\varepsilon, p} \rightarrow T V_{\varepsilon, p}$ only vanishes at the point $p=0$. The group $\Gamma_{p, g}$ acts on $T V_{\varepsilon, 0}$ through the standard action of $\mathrm{O}(n, \mathbb{R})$ on $\mathbb{R}^{n}$. Let $\rho_{p, g}: \Gamma_{p, g} \rightarrow \mathrm{O}(n, \mathbb{R})$ be the corresponding representation.

We know from Lemma 2.1 that the class $\left[\left(\mathscr{H}_{p, g}, P_{p, g}\right)\right] \in \mathrm{R}\left(\Gamma_{p, g}\right)$ only depends on the homotopy class of $\theta_{p, g}$, where, by Lemma 2.2 we may restrict $\theta_{p, g}$ to arbitrarily small open balls around 0 .

Note first that under the identification of $V_{\varepsilon, p}$ with a neighborhood of 0 in $\mathbb{R}^{n}$ via the exponential map, the metric, call it $\rho$, on $V_{\varepsilon, p}$ is not necessarily the flat metric coming from $\mathbb{R}^{n}$. However the convex combination of the metric $\rho$ and the Euclidean metric, $\rho_{\mathbb{R}^{n}}$, gives a homotopy, $\rho_{t}$, between these two metrics. That, in turn, gives a homotopy, $\left.\left.\theta_{t}:=\frac{\rho_{t}}{\varepsilon}\left(d_{y} \rho_{t}\right)\right)(\phi(g x), x)\right)$ of corresponding vector fields. Therefore without loss of generality, we may assume that the set $V_{\varepsilon, p}$ is equipped with the Euclidean metric and that $\theta_{p, g}(x)=x-(\phi \circ g)(x)$. By calculus, $x-(\phi \circ g)(x)=$ $\left(\operatorname{Id}_{\mathbb{R}^{n}}-d(\phi \circ g)(p)\right) \cdot x+\psi(x)$ for some $\psi$ such that $\frac{\psi(x)}{\|x\|} \rightarrow 0$ as $x \rightarrow 0$. Then, in a small neighbourhood of $p=0, t \mapsto\left(\operatorname{Id}_{\mathbb{R}^{n}}-d(\phi \circ g)(p)\right) \cdot x+t \psi(x)$ gives a homotopy, $\theta_{p, g} \sim\left(\operatorname{Id}_{\mathbb{R}^{n}}-d(\phi \circ g)(p)\right) \cdot x=W_{p, g} X$ with $W_{p, g}=\operatorname{Id}_{\mathbb{R}^{n}}-d(\phi \circ g)(p)$. It follows then from Lemma 2.5 together with Corollary 2.7 that

$$
\operatorname{ind}\left(Q_{p, g}\right)=\frac{1}{\left|\Gamma_{p, g}\right|} \sum_{g \in \Gamma_{p g}} \chi\left(\rho_{p, g}, W_{p, g}\right)(g),
$$

where $\chi(\rho, A)(h)=\operatorname{sign} \operatorname{det}\left(\left.A\right|_{\mathrm{Fix}(h)}\right)$ is the orientation character as in Definition 2.4.

Putting all together, we have the following theorem: 
Theorem 3.7. The pairing $\langle[\hat{\alpha}], \Delta\rangle$ is given by

$$
\langle[\hat{\alpha}], \Delta\rangle=\sum_{(p, g) \in \Sigma} \frac{1}{\left|\Gamma_{p, g}\right|} \sum_{h \in \Gamma_{p, g}} \chi_{\left(\rho_{p, g}, W_{p, g}\right)}(h) .
$$

The above theorem together with the abstract Lefschetz theorem of [9] proves our Lefschetz fixed point theorem, Theorem 0.1.

We now discuss an example.

Example 3.8. Let $G \cong \mathbb{Z} \rtimes \mathbb{Z} / 2 \mathbb{Z}$ be the infinite dihedral group. It is the subgroup of Iso $(\mathbb{R})$ generated by $u(x)=-x$ and $w(x)=x+1$. It has the relation $u w u=w^{-1}$, and has two conjugacy classes of finite subgroups $K_{1}:=\langle u\rangle=\operatorname{Stab}_{G}(0)$, and $K_{2}:=\langle w u\rangle=\operatorname{Stab}_{G}\left(\frac{1}{2}\right)$. A fundamental domain for the action is the interval $\left[0, \frac{1}{2}\right]$. Note that $\dot{0} \neq \frac{\dot{i}}{2} \in \dot{\mathbb{R}}$, where we use dot notation to indicate orbits.

The K-theory of $C_{0}(\mathbb{R}) \rtimes G$ is $\mathbb{Z}^{3}$ in dimension 0 and is trivial in dimension 1 . A general property of proper actions tells us that $C(G \backslash \mathbb{R})=C[0,1]$ is strongly Morita equivalent to an ideal in $C_{0}(\mathbb{R}) \rtimes G$, and one K-theory generator corresponds under this strong Morita equivalence and the inclusion of the ideal, to the class of the unit in $C(G \backslash \mathbb{R})$. We denote this class $[E]$. The other two projections come from the $C^{*}\left(K_{i}\right), i=1,2$. We denote them $\left[p_{i}\right], i=1,2$.

Let

$$
\phi: \mathbb{R} \rightarrow \mathbb{R}, \quad \phi(x)=-x-\frac{1}{2} .
$$

Let $\zeta: G \rightarrow G$ be $\zeta(u)=u w$ and $\zeta(w)=w^{-1}$. Then $\zeta$ extends to an automorphism of $G$, and $\phi(\zeta(x))=g \phi(x)$ is easily checked for $g=w, u$, so that we get a covariant pair. The map $\phi$ has one fixed orbit, which is $\frac{i}{4}$; note that $\phi$ itself fixes $\frac{1}{4}$. The derivative at this point is -1 , so that we get a positive sign attached to this point. Since $\frac{i}{4}$ has no isotropy in $G$, we only get a contribution of +1 from this fixed orbit: the local side of the Lefschetz formula is equal to 1 . On the global side, since $\zeta\left(K_{1}\right)=$ $K_{2}$, there is no tracial contribution from the summands $\mathbb{Z} p_{1} \oplus \mathbb{Z} p_{2}$, and therefore $\operatorname{trace}_{s}\left(\alpha_{*}\right)=1$, with $\alpha: C_{0}(\mathbb{R}) \rtimes G \rightarrow C_{0}(\mathbb{R}) \rtimes G$ the induced automorphism.

For a second example, let $\zeta$ be the identity. Let $\phi$ be a small perturbation of the identity map $\mathbb{R} \rightarrow \mathbb{R}$ which can be roughly described as follows. Firstly, $\phi$ maps the interval $\left[0, \frac{1}{2}\right]$ to itself. It fixes 0 and $\frac{1}{2}$, and has derivative zero at both these points. It also fixes the point $\frac{1}{4}$, and has derivative rather large at this point (in particular greater than 1). Finally, $\phi$ is extended to a $G$-equivariant map $\mathbb{R} \rightarrow \mathbb{R}$ in the obvious way.

Clearly $\phi$ is proper $G$-homotopic to the identity, so its graded trace on K-theory is 3. It has three fixed orbits $\dot{0}, \frac{\dot{i}}{4}$, and $\frac{\dot{i}}{2}$, which are actually fixed points in $\mathbb{R}$. The first and third of these come with a positive sign, and are weighted by the number of conjugacy classes (i.e., the number of elements) in the isotropy groups $K_{1}$ and $K_{2}$ of these points. We thus get a contribution of $(1+1)+(1+1)=4$ from the first and third fixed points, and, since $\frac{i}{4}$ has no isotropy and $\phi^{\prime}\left(\frac{1}{4}\right)>1$, we get a contribution of -1 from the second fixed point, with a net contribution of 3 , as required. 
On the other hand, if we change the above map $\phi$ just to have now large derivatives at 0 and $\frac{1}{2}$ and zero derivative at $\frac{1}{4}$. Then we get a contribution $0+1$ from 0 and also the same from $\frac{1}{2}$, and 1 from $\frac{1}{4}$, with a net contribution of 3 again.

\section{References}

[1] M. F. Atiyah and I. M. Singer, The index of elliptic operators: I. Ann. of Math. (2) 87 (1968), 484-530. Zbl 0164.24001 MR 0236950

[2] M. F. Atiyah and G. B. Segal, The index of elliptic operators: II. Ann. of Math. (2) 87 (1968), 531-545. Zbl 0164.24201 MR 0236951

[3] S. Baaj and P. Julg, Théorie bivariante de Kasparov et opérateurs non bornés dans les $C^{*}$-modules hilbertiens. C. R. Acad. Sci. Paris Sér. I Math. 296 (1983), 875-878. Zbl 0551.46041 MR 715325

[4] P. Baum, A. Connes, and N. Higson, Classifying space for proper actions and $K$-theory of group $C^{*}$-algebras. In $C^{*}$-algebras: $1943-1993$ (San Antonio, TX, 1993), Contemp. Math. 167, Amer. Math. Soc., Providence, RI, 1994, 241-291. Zbl 0830.46061 MR 1292018

[5] B. Blackadar, K-theory for operator algebras. Math. Sci. Res. Inst. Publ. 5, 2nd ed., Cambridge University Press, Cambridge 1998. Zbl 0913.46054 MR 1656031

[6] S. Echterhoff, H. Emerson, and H. J. Kim, KK-theoretic duality for proper twisted actions. Math. Ann. 340 (2008), 839-873. Zbl 1147.19006 MR 2372740

[7] H. Emerson, Noncommutative Poincaré duality for boundary actions of hyperbolic groups. J. Reine Angew. Math. 564 (2003), 1-33. Zbl 1040.46048 MR 2021032

[8] H. Emerson, The Baum-Connes conjecture, noncommutative Poincaré duality, and the boundary of the free group. Internat. J. Math. Math. Sci. 2003 (2003), Issue 38, 2425-2445. Zbl 1037.46059 MR 1995725

[9] H. Emerson, Lefschetz numbers for $C^{*}$-algebras. Preprint 2007. arXiv:0708.4278

[10] H. Emerson and R. Meyer, Euler characteristics and Gysin sequences for group actions on boundaries. Math. Ann. 334 (2006), 853-904. Zbl 1092.19003 MR 2209260

[11] H. Emerson and R. Meyer, A descent principle for the Dirac-dual-Dirac method. Topology 46 (2007), 185-209. Zbl 1119.19005 MR 2313071

[12] C. Farsi, K-theoretical index theorems for orbifolds. Quart. J. Math. Oxford Ser. (2) 43 (1992), 183-200. Zbl 0768.58049 MR 1164622

[13] N. Higson and J. Roe, Analytic K-homology. Oxford Math. Monogr., Oxford University Press, Oxford 2000. Zbl 0968.46058 MR 1817560

[14] G. G. Kasparov, Topological invariants of elliptic operators. I: K-homology. Izv. Akad. Nauk SSSR Ser. Mat. 39 (1975), 796-838; English transl. Math. USSR-Izv. 9 (1975), 751-792. Zbl 0337.58006 MR 0488027

[15] G. G. Kasparov, The operator $K$-functor and extensions of $C^{*}$-algebras. Izv. Akad. Nauk SSSR Ser. Mat. 44 (1980), 571-636; English transl. Math. USSR-Izv. 16 (1981), 513-572. Zbl 0464.46054 MR 582160 
[16] Equivariant $K K$-theory and the Novikov conjecture. Invent. Math. 91 (1988), 147-201. Zbl 0647.46053 MR 918241

[17] G. G. Kasparov, $K$-theory, group $C^{*}$-algebras, and higher signatures (conspectus). In Novikov conjectures, index theorems and rigidity, Vol. 1 (Oberwolfach, 1993), London Math. Soc. Lecture Note Ser. 226, Cambridge Univ. Press, Cambridge 1995, 101-146. Zbl 0957.58020 MR 1388299

[18] G. Kasparov and G. Skandalis, Groups acting properly on "bolic" spaces and the Novikov conjecture. Ann. of Math. (2) 158 (2003), 165-206. Zbl 1029.19003 MR 1998480

[19] D. Kucerovsky, The $K K$-product of unbounded modules. $K$-Theory 11 (1997), 17-34. Zbl 0871.19004 MR 1435704

[20] W. Lück and J. Rosenberg, Equivariant Euler characteristics and $K$-homology Euler classes for proper cocompact $G$-manifolds. Geom. Topol. 7 (2003), 569-613. Zbl 1034.19003 MR 2026542

[21] G. Segal, Equivariant $K$-theory. Inst. Hautes Études Sci. Publ. Math. 34 (1968), 129-151. Zbl 0199.26202 MR 0234452

Received June 16, 2008; revised January 4, 2009

S. Echterhoff, Westfälische Wilhelms-Universität Münster, Mathematisches Institut, Einsteinstr. 62, 48149 Münster, Germany

E-mail: echters@uni-muenster.de

H. Emerson, Department of Mathematics and Statistics, University of Victoria, PO BOX 3060 STN CSC, Victoria, B.C., Canada V8W 3R4

E-mail: hemerson@math.uvic.ca

H. J. Kim, Department of Mathematics and Statistics, University of Victoria, PO BOX 3060 STN CSC, Victoria, B.C., Canada V8W 3R4

E-mail: hjeong99@gmail.com 